\title{
Managing high-end ex-demonstration product returns
}

\author{
L. Muyldermans * \\ Nottingham University Business School, \\ Jubilee Campus, Wollaton Road, \\ Nottingham, NG8 1BB, UK \\ Luc.Muyldermans@nottingham.ac.uk \\ L. N. Van Wassenhove \\ INSEAD \\ Blvd de Constance \\ 77305 Fontainbleau Cedex, France \\ Luk.Van-Wassenhove@insead.edu \\ V. D. R. Guide, Jr. \\ Department of Supply Chains \& Information Systems \\ Smeal College of Business Administration \\ The Pennsylvania State University \\ University Park, PA 16802 USA \\ dguide@psu.edu
}

\begin{abstract}
:
Some manufacturers demonstrate their products so that customers can gain experience before making a purchase. We present a novel application of a closed-loop supply chain where product returns from demonstrations of high-end IT equipment are substantial and the major delay in the system is due to the long demonstration time at the client sites. In addition, the product lifecycle is short and the value erodes rapidly over time, with steep drops in the resale revenue when new product generations are introduced. We present a finite lifecycle model that captures the key trade-offs in this environment, that is, either to reuse a collected ex-demo product for a next demonstration or to salvage its residual value in the secondary market and use a new product to satisfy the next demo request. We derive two cost/revenue signals that enable us to distinguish between fast and slow value erosion. We show that the fast/slow erosion decision is dynamic and depends on the rate of value erosion and the length of the demonstration time. We analyze the optimal demo pool strategies and show that in the case of fast erosion it may be better to postpone reuse activities until later in the lifecycle. We illustrate our model using empirical data from a large IT manufacturer and formulate several guidelines so as to better manage high value ex-demonstration product returns.
\end{abstract}

Keywords: Supply Chain Management, Reuse closed-loop supply chains, Ex-demonstration product returns, Time-sensitivity, Continuous time transportation problem application

(* Corresponding author) 


\section{Introduction}

Manufacturers and retailers often demonstrate their products. Through these demonstrations customers gain experience before making a purchase and reduce their uncertainty about the product's performance and its ability to match their needs (Heiman et al., 2001). Surprisingly, there is limited empirical evidence, or theoretical research, demonstrating the net monetary effects of demonstrations. In many cases, the manufacturer's financial risk of providing demo products may be limited. This is true when the demonstration cost is low (e.g., downloadable software), or when the demonstration time is short compared with the product lifecycle so that products can be reused in repeat presentations or tests (e.g., car test drives). For expensive, high-end, customized products such as high-end IT, audio \& music, optical, medical and industrial equipment, the demonstration time is high. These products are "time products' in Heiman and Muller's (1996) classification matrix, because the desirable and undesirable product characteristics are revealed slowly. Hence, the manufacturer's financial risk to demonstrating these products may be much higher.

In this paper we consider product demonstrations of the last category, and we look at the demo services from a closed-loop supply chain perspective. Closed-loop supply chains (Guide and Van Wassenhove, 2003; Guide et al., 2003; Dekker et al., 2004; Guide and Van Wassenhove, 2009) are supply chains designed and managed to explicitly account for return streams in the forward supply chain operations over the entire product lifecycle. The area of reverse logistics and closed-loop supply chain management has evolved into a very rich research domain (for overviews, see e.g., Sasikumar and Kannan, 2009; Govidan et al. 2015; Govidan and Soleimani, 2017). In our application, a closed-loop is formed by reusing demonstration equipment repeatedly before the equipment is retired and leaves the system. Many other studies of reuse closed-loop supply chains can be found in the literature (e.g., Kroon and Vrijens, 1995; Krikke et al., 1999; Geyer and Jackson, 2004; Krikke et al., 2004; Geyer et al., 2007; Östlin et al., 2008; Atasu et al., 2008) but ex-demonstration product returns have not been adequately addressed in previous research.

A key question in our problem involves deciding what to do with ex-demo products. Returns from demonstrations can be substantial and may tie up large amounts of capital high-end products. For instance, a large manufacturer of high-end IT equipment that the authors investigated can sell refurbished ex-demo equipment in the secondary market. Another, less preferred, option is to recycle and scrap the products. Due to the nature of such products (technology equipment) and their short lifecycles, the resale revenue decreases rapidly over time. There is significant pressure to keep system delays short so as to enable more reuse and/or increase resale revenue.

The importance of the marginal value of time in forward supply chains is well established (Blackburn, 1991). Only more recently has the value of time (value erosion or value decay) been investigated in relation to product returns management (e.g., Blackburn et al., 2004; Guide et al., 2005; Guide et al. 2006; Guide et al., 2008; Ruiz-Benítez et al, 2014; Pazoki and Abdul-Kader, 2016; Kadanbala et al., 2017 and Difrancesco et al, 2018). These studies have focused on different aspects 
and influences of time-value on strategic, structural or operational decisions in the reverse supply chain, and have also applied different modelling approaches. Atasu and Çetinkaya (2006) and Ruiz-Benítez et al. (2014) focus on inventory control policies to coordinate collections of returns between a collection point and a remanufacturer. Guide et al. (2006) and Difrancesco et al. (2018) apply queuing network models and investigate the impact of system delays and value erosion for different reverse supply chain designs (Guide et al., 2006)), or to gain insight in return window decisions for online fashion retailers (Difrancesco et al., 2018). Queuing theory based models are well-suited to deal with system delays and different value decay rates (typically, modelled through exponential functions), yet they focus on steady state behavior so that dynamic aspects (e.g., time varying demand and decisions) are more difficult to account for. These models are also descriptive in nature: it is up to the decision modeler to propose and experiment with various alternatives. Discrete-time, multiple period linear (LP) and integer programming (IP) models have also been employed (e.g., Guide et al., (2005); Fang et al. (2016)). These models do have the capability to deal with various time varying features (costs, demands and decisions), but they are less flexible in their ability to deal with delays (lead times and delays must be integer multiples of the base planning period). With LP and IP models it may also be more difficult to uncover more general insights, especially if conclusions are to be derived from a rather limited set of computational experiments.

A key difference between the previous studies and the study we present here is that we consider a finite lifecycle model (see e.g., Atasu and Çetinkaya (2006) and Geyer et al. (2007)). This is of particular importance for high-end equipment (e.g. IT, medical and industrial applications) considered here where the product lifecycle is short and the number of reuse cycles are limited. We investigate this problem in continuous time and we model it as special type of a Separated Continuous Linear Program (SCLP) (see e.g., Pullan (1996)), which we can convert (as shown in Appendix) into a Continuous time Transportation Problem (CTP). To the best of our knowledge, we are not aware of other SCLP applications in the area of reverse logistics. SCLP is an elegant modelling approach, especially for dynamic problems. These models, however, are less popular than their approximate multiple period linear programming analogues, derived by time discretization. The solution approach we present (as well as to SCLPs more generally, see e.g. Pullan (1996)) changes at discrete points in time over the planning horizon. Our attention is devoted to uncover and explain where and why these switch points occur in the product lifecycle. This analysis enables stronger and more generalizable conclusions compared to analyzing our problem in discrete time.

We use a case example in Section 2 to describe and analyze the generic features of a demonstration service environment for high-end equipment that needs to be captured in a decision model. Section 3 presents a generic, deterministic, continuous time, finite lifecycle model that captures the key trade-offs in the high-end equipment environment where a demonstration product is collected and reused for a further demo request, or its residual value is salvaged in the secondary market. The objective is to maximize the revenue from selling ex-demo products in the secondary market while 
making sure that all demo requests are met at minimum cost. Value erosion is modelled as a nonincreasing secondary market revenue function. We demonstrate that a zero-inventory policy holds and that the model can be transformed into a Continuous time Transportation Problem. We derive two cost/revenue signals that enable us to distinguish between fast and slow value erosion. We show that the fast/slow erosion decision is dynamic and depends on the rate of value erosion and the length of the demonstration time. Sections 4 and 5 present the optimal demonstration pool strategies for both slow and fast erosion respectively. The focus in our analysis is on identifying the different time epochs over the product lifecycle where the solution structure changes. We show that in the case of fast erosion, it may be better to postpone the reuse activities until later in the lifecycle even if this means that more exdemonstration equipment will be scrapped later. In Section 6, we illustrate the application of the model to derive the optimal strategy for an example, and show that for a specific case company substantial savings can be made. The model and modelling approach has applicability for the design and management of closed-loop supply chains for high-end equipment and machinery products that are demonstrated before sale. Our conclusions and areas for further research follow in Section 7.

\section{Product demonstrations at company $X$}

Company X's European Demo Service Center (or demo store) is co-located with the factory where new and remanufactured IT products are produced. The company's product portfolio includes high-end, mid-range and entry-level servers, network storage systems and industry standard servers. The demo store provides and manages demonstration equipment for sales and marketing support activities including participations in exhibitions and tradeshows, demonstrations and evaluations at resellers and end-customers, assignments to demo rooms or benchmark centers, and internal training of the sales force. Company $\mathrm{X}$ generates, in Europe, revenues in excess of $\$ 200 \mathrm{M}$ each year from selling remanufactured products. About $65 \%$ of these products involved ex-demonstration equipment, $20 \%$ were customer and partner returns and $15 \%$ were inventory excesses. All these products were less than three years old, but involved up to five generations of technology.

Figure 1 schematically presents the product flows through the demo service center. New products and components are ordered in the store (arc 1) based on demo requests by sales representatives for potential customers. Typically, each demo is customized and built to match specific customer needs. The complete demo solution may comprise products from different product lines, e.g., an IT server solution in combination with a storage system. When products arrive in the store, they are forwarded and installed at the demo site ( $\operatorname{arc} 2)$ where the client tests the equipment for a certain time - the demo loan period (in this case a maximum 3 months). During the loan period the client may decide to purchase the equipment, in which case the equipment leaves the system (buyout) (arc 3). Alternatively, the demo is collected from the client and returned to the store ( $\operatorname{arc} 4)$. A demo returned at the store can be reused for a second or a third installation at a client site, or it can be retired and sent to the returns center (arc 5). Here it is disassembled, tested, refurbished and sold in the secondary 
market (arc 6); or scrapped and recycled. The returns center also receives product returns from outside the demo process $(\operatorname{arc} 7)$. These products are handled in the returns center in a similar way. Finally, it is also possible to order products from the returns center into the demo store (arc 8) and use these (instead of new products) to satisfy demo requests.

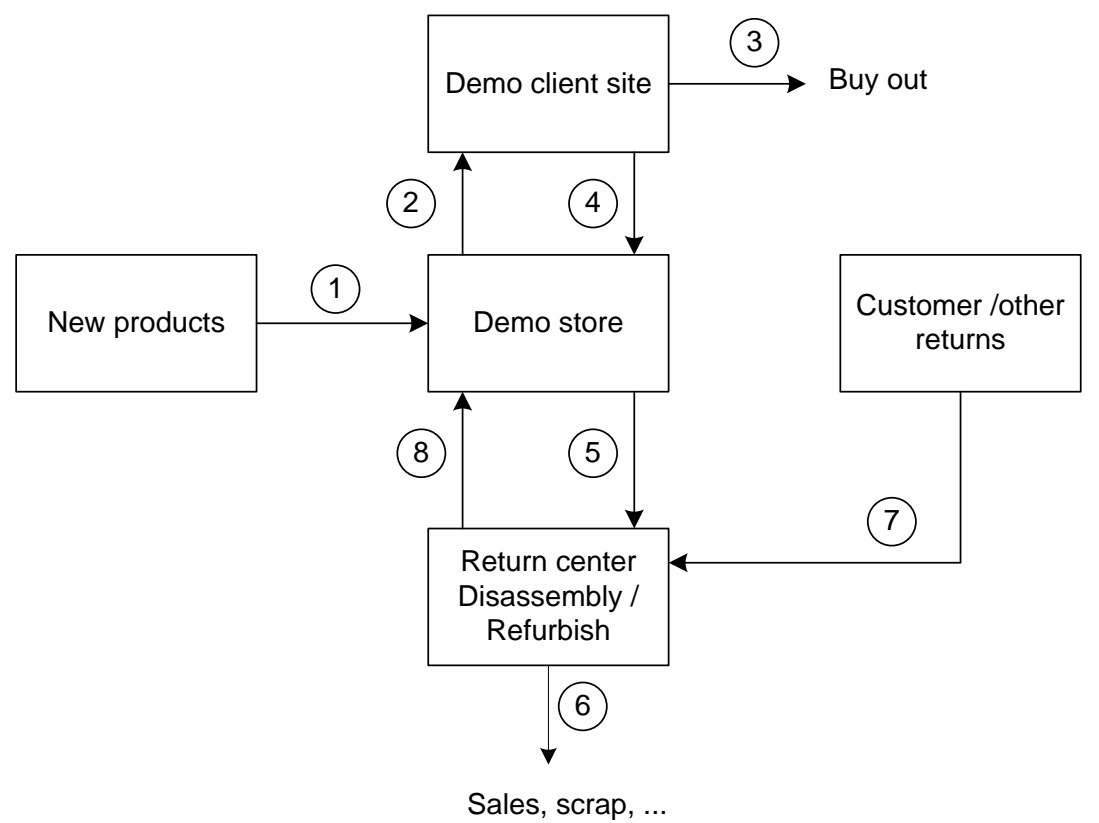

Figure 1: The demo process - a closed-loop supply chain

The demo pool managers at company $\mathrm{X}$ focused on the technical aspects of the equipment, rather than taking a supply chain perspective and looking at the entire system in terms of flows and bottlenecks. The demo process was controlled by a set of simple rules (such as maximum loan period and maximum residence time of a product in the demo process before retirement), with the intention to use each newly ordered product in two or three demo installations.

We analyzed the flows (as shown in Figure 1) for a selection of mid-range servers. Different server configurations may exist, but their cost and price are mainly determined by a few core components (processor, server base unit, memory modules), which is typical in many types of high-end equipment. The commonality between servers is high when we focus on the core components. The analysis below concentrates on the server processors. These are a key component in every server; they are expensive (up to several $\$ 10,000$ ), and are lifecycle defining. New generations of processors are introduced into the market every nine to ten months. A server's sales price erodes constantly over time (values of 1-3\% per month are not uncommon), and when new generations of processors are introduced, the sales price of the older technology drops sharply.

Figure 2 shows the demand and pricing information for a specific processor (processor 1) over its lifecycle (we note that the numbers have been disguised). The profile of the new products demand curve is almost triangular. The profile of the demo requests curve exhibits a very similar pattern with a (less pronounced) peak near October-November 2015. In this case the demo services seem well 
coordinated with the new product sales. The third curve, near the base of the graph, corresponds to the sales of refurbished products in the secondary market. These volumes are much lower and lag (typically 12 to 15 months) the shipments to the primary market. This may be due to the limited availability of returned and refurbished products earlier in the lifecycle, or an undeveloped secondary market, or a strategic decision regarding when to start selling in the secondary market so as to avoid cannibalization effects on new product sales.
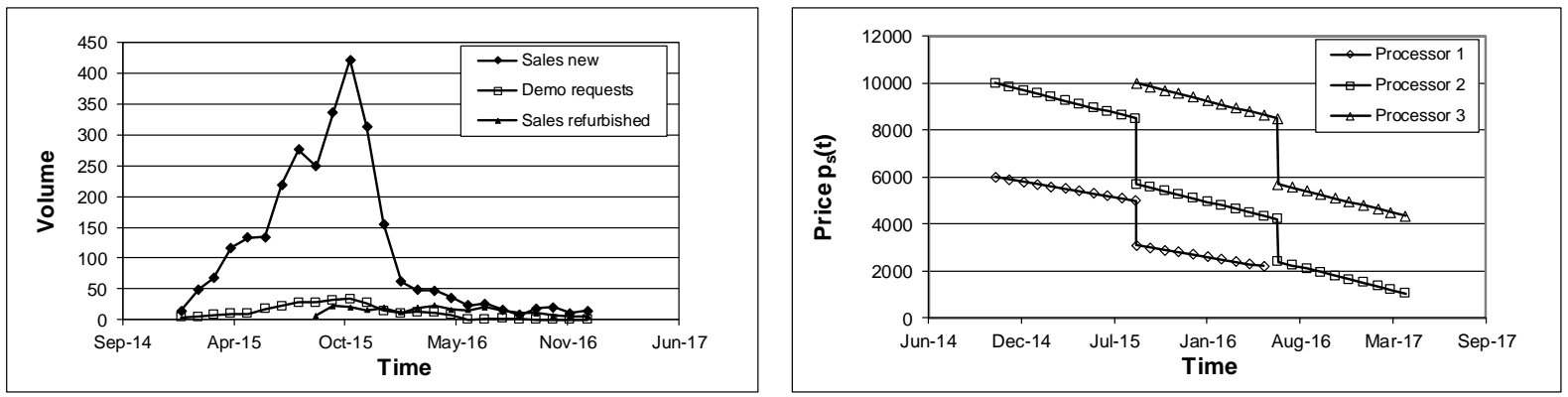

Figure 2: Demand and price of processor 1 over its lifecycle

The curves on the right in Figure 2 show typical processor sales prices over their lifecycle. Processors 1 and 2 were introduced in the market in November 2014. Processor 2 is more powerful, hence the higher price. In August 2015, a new generation processor became available, and consequently the prices for both processors 1 and 2 dropped considerably. Processor 1's active lifecycle finishes around August 2016, while processor 2's lifecycle continues for a few more months.

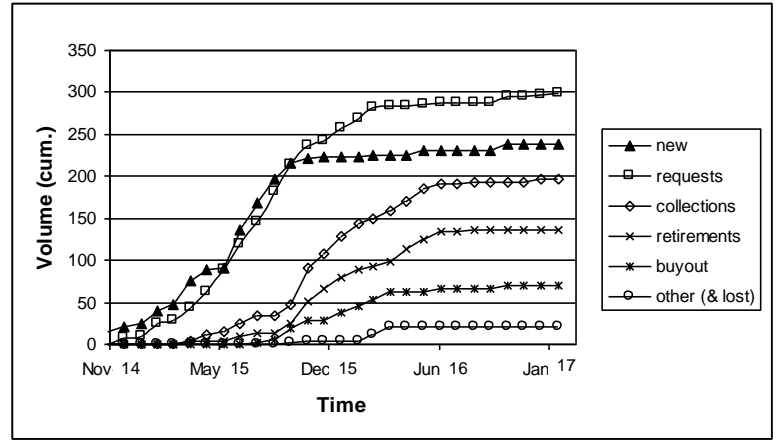

A: processor 1

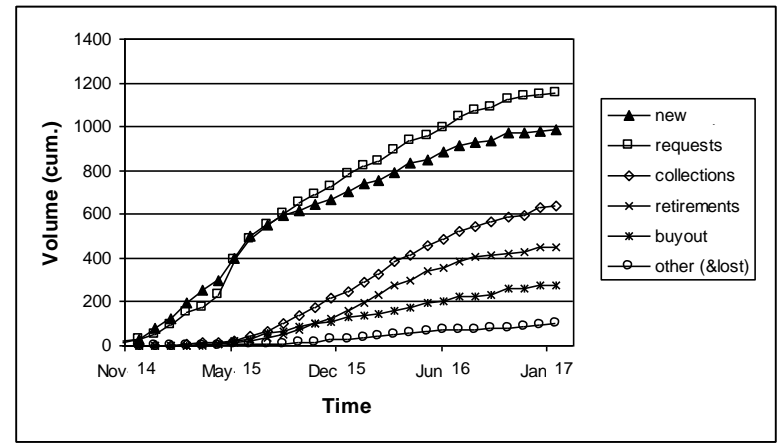

B: processor 2

Figure 3: Demo flow analysis for two processors

Figure 3 presents in more detail the different flows in the demo process for processors 1 and 2. The curves show the cumulative volumes over time of demo requests ( $\operatorname{arc} 2$ in Figure 1); new processors ordered in the store (arc 1 in Figure 1); collections from the client sites back to the store (arc 4 in Figure 1); retirements to the returns center (arc 5 in Figure 1); and buyouts ( $\operatorname{arc} 3$ in Figure 1). We note that all the demo requests in Figure 3 are satisfied: the earliest requests are always fulfilled by using new products (the new products curve in Figure 3 almost coincides with the requests curve). For processor 1, most requests after August 2015 are satisfied by reusing earlier collected products (the 'new' curve 
is rather flat, but the requests curve is still increasing). The horizontal displacement between the requests curve and the collections curve (adjusted for buyouts) is an indication for the actual demo loan time. The actual time that the product stayed at the clients is several months, and usually much longer than the maximum target of three months. Processor 2 is demonstrated over a longer period and most of its requests are satisfied by ordering new products in the store, even when collected / retired products were available. The buy-out rates are low and rather insignificant before August 2015.

Table 1 summarizes for 5 different processors a number of key measures. (Note that not all processors had reached end-of-life when the data were collected. The figures are total volumes over a time period starting at each product's launch date until the time of data collection.)

Table 1: Key measures of 5 processors used in demonstrations

\begin{tabular}{lccccc}
\hline & Processor 1 & Processor 2 & Processor 3 & Processor 4 & Processor 5 \\
\hline $\begin{array}{l}\text { Total demo requests } \\
\text { New products ordered in } \\
\text { the store }\end{array}$ & 298 & 1155 & 641 & 723 & 750 \\
$\begin{array}{l}\text { Buy out } \\
\begin{array}{l}\text { Units still in the system } \\
\text { (+ others) }\end{array}\end{array}$ & 33 & 986 & 554 & 633 & 669 \\
$\begin{array}{l}\text { Average loan period } \\
\text { (days) }\end{array}$ & 132 & 279 & 134 & 144 & 86 \\
$\begin{array}{l}\text { Demo returns } \\
\text { (retirements) }\end{array}$ & 136 & 450 & 307 & 159 & 366 \\
$\begin{array}{l}\text { Other returns (outside the } \\
\text { demo process) }\end{array}$ & 101 & 159 & 113 & 342 & 217 \\
$\begin{array}{l}\text { Total returns } \\
\begin{array}{l}\text { Sales 2 } \\
\text { and other returns) }\end{array}\end{array}$ & 237 & 609 & 132 & 673 & 317 \\
\begin{tabular}{l} 
Sales 1 $1^{\text {st }}$ Market \\
\hline
\end{tabular} & 2836 & 544 & 117 & 467 & 132 \\
\hline
\end{tabular}

The following conclusions are apparent from Table 1. Most demo requests (about 85\%) are satisfied by new products. The buyout rate (as a percentage of the total requests) is low (about 20\%). With respect to the loan period, only processor 1 seems under control. For the others, the average loan period is above the maximum target of 3 months. The returns from the demo process make up a significant fraction of the total returns at the returns center, typically more than $65 \%$. This ratio demo returns/other returns may decline over time. Most demo returns arrive in the second half and near the end of the active lifecycle of the processor. When new processor generations are introduced, clients can upgrade their servers, or trade-up the older processors for newer technology. Upgrade and trade-up programs generate a second stream of returns, which may arrive much later, long after the active lifecycle of the old processor has finished. These products have lost most of their value so that re-introduction in the secondary market is almost impossible. (In Table 1, the 'other returns' for processor 4 are much higher 
compared with other processors, and a significant fraction of these was due to upgrade and trade-up campaigns). The last two rows show the volumes sold in the secondary market (refurbished products) and in the primary market (new product sales). Company X manages to sell most of its demo returns in the secondary market, but there is a practice of delaying and spreading out sales over a rather long period (see Figure 2) to avoid cannibalization with new product sales.

Effective demo pool management is not an easy task. There are several characteristics and control parameters which may affect the overall profitability or cost effectiveness of the operations. These include the shape of the demo request profile over the product's lifecycle, the demo loan period, the buyout rate, the cost of new products, the sales price of refurbished products in the secondary market and its erosion over time, the time window in which to sell refurbished products, the secondary market capacity or potential, and the availability of other returns which can be used for demonstration. Company $\mathrm{X}$ used simple decision rules to control the demo store. These rules may work well under certain conditions but may need modification or refinement in other situations.

Our aim in this paper is to develop a model that captures the core aspects of a generic class of high-end equipment problems that require demonstration as part of the sales process, and to derive optimal policies for effective decision making in terms of reuse and/or resale in the secondary market of ex-demonstration product returns. As in Guide et al., (2005) by discretizing the lifecycle or planning horizon into time buckets, multiple-period linear programming models that incorporate all the abovementioned features can be developed. LP models are easy to solve and flexible in terms of handling various extensions. On the other hand, it is difficult to obtain general insights through experimentation with LPs. Therefore, we investigate the high-end equipment demonstration problem in continuous time, focusing our attention on the cost/profitability signals that drive the optimal solution and the moments in time in the planning horizon when the solution structure changes. We present our model together with the assumptions in Section 3.

\section{Model, assumptions and notation}

The problem environment modelled is shown in Figure 4. We assume that the requests $d(t)$ for demos at any time $t$ are known and occur over a finite lifecycle of length $T$, and that the demand profile is concave (or unimodal i.e., monotonically increasing for $t \leq m$ and monotonically decreasing for $t \geq m$, with maximum at $m$ ) over $[0, T)$. A concave, or unimodal, demand profile is a good approximation for short lifecycle products (such as IT equipment) and has also been used in previous research (e.g., Atasu and Çetinkaya (2006) and Geyer et al. (2007)). The requests must be satisfied at the time when they occur, either by new products ordered from a supplier $\left(\operatorname{cost} c_{N}\right)$, or by reusing previously collected and refurbished demos. The demo loan period $L$ (residence time at the client site) is assumed to be constant and is the only major delay in the system. That is, the time to order a product from the supplier, the time to transport/collect a demo product to/from a client site and the time for refurbishment are negligible (in practice, a few days at most) compared with the demo loan period (typically several 
months). It is possible to specify a buyout profile, but this only results in a modification of the original demo requests profile. It is therefore not incorporated in the model and all demo products are collected after loan period $L$, with a time-phased collection profile $d(t-L)$ for $L \leq t<T+L$.

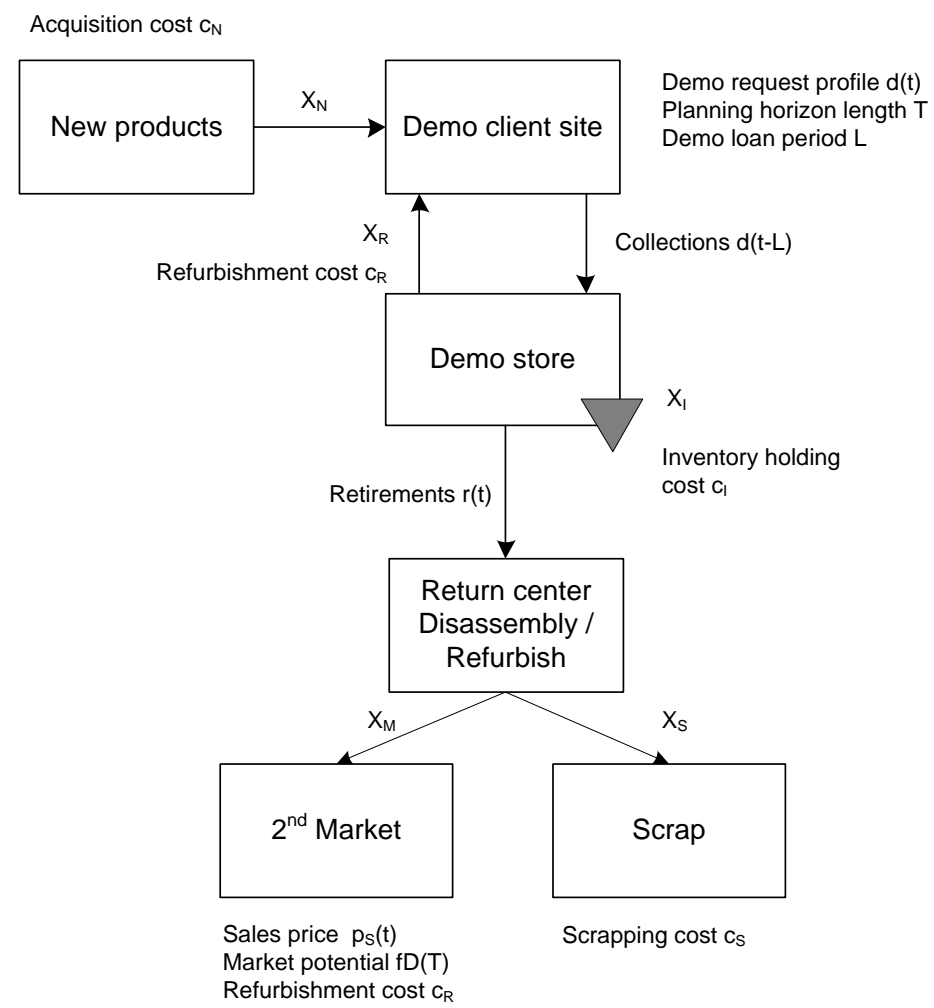

Figure 4: Problem environment modelled.

There is no starting inventory of new products in the demo system: new products are ordered from the supplier when these are required to fulfil a request. For high-tech customized equipment demos are built-to-order after assessing the customer's needs, and the store does not stock new products because these are too expensive. The new products node in Figure 4 corresponds to a source (supplier) that can provide new products whenever required. Collected ex-demo products enter the returned products inventory $\left(\operatorname{cost} c_{I}\right)$ at the demo store and can be refurbished $\left(\operatorname{cost} c_{R}\right)$ and reused for a next demo request. Alternatively, collected ex-demo equipment can retire from the demo store and either be sold in the secondary market (after refurbishment), or scrapped (cost $c_{S}$ ). The scrapping cost can be negative and represents a (typically small) salvage value received from selling the retired equipment to a recycling vendor or scrap broker when the option to sell in the secondary market is exhausted. At the beginning of planning period there is no inventory of collected ex-demo products in the store.

In line with our application context of high-end customized products, we also assume all costs $\left(c_{N}, c_{R}, c_{I}\right.$ and $\left.c_{S}\right)$ to be constant. These are valid assumptions since the product lifecycle is short. We could easily incorporate transportation and collection costs, but these are very low in comparison with the dominant cost in our application (the new product acquisition cost $c_{N}$ ). In fact, because all demo requests have to be satisfied and collected, and because there is no difference in transportation and collection cost depending on whether new or refurbished products are used, both transportation and 
collection cost can be considered as sunk cost - incorporating these does not affect the optimal demo policy. We also note that the new product $\operatorname{cost} c_{N}$ is high compared with the refurbishment $\operatorname{cost} c_{R}$ and scrap cost $c_{S}$. This is due to the nature of the returns. Almost all ex-demo products have been used over a rather limited time only (a few months) and are collected in excellent or like-new condition. The refurbishment operations are low-touch, i.e., inspecting, testing and dis- and re-assemble (modular) demo equipment. There is no hard repair involved.

Apart from reuse, the main value recovery mechanism in this problem environment is through sales in the secondary market. We account for value erosion or price decay in the secondary market. We assume that the secondary market sales price erodes over time from the launch of the product (time $t=0$ ), until the end of the planning horizon $T+L$. Retired demos are sold in the secondary market at time $t$ and fetch a price $p_{s}(t)$. We do not pose strong restrictions on the revenue function: we only assume that $p_{s}(t)$ is piecewise analytical and that it is non-increasing over time (i.e., $p_{s}\left(t_{1}\right) \geq p_{s}\left(t_{2}\right)$ for $t_{1}$ $\left.\leq t_{2}\right)$. Piecewise analytical means that $p_{s}(t)$ may have discontinuities: $p_{s}(t)$ is right-continuous but not necessarily left-continuous. Our results remain valid for any erosion function that fits these conditions (including continuous, linear and exponential erosion, which are used in many studies). As in most studies, in our model only revenue is affected by time. Some studies also address erosion in other components (e.g., Guide et al. (2006) account for erosion in production and remanufacturing cost) and/or discount cash in/out flows (Guide et al. (2006) and Difrancesco et al. (2018)). We have not incorporated a discount factor in this model because all cash in/out flows happen over a short lifecycle only. Our model can be extended to deal with more time varying features and in Section 7, we comment on how our results are affected in the case where cash flows are discounted.

The demand potential in the secondary market is further limited by a global market constraint or capacity, but there is no restriction (apart from the availability of ex-demo equipment) on when sales can start or when sales must end.

The objective in the model is to maximize revenue gained from selling ex-demonstration products in the secondary market, whilst making sure that all demonstration requests are satisfied at minimum cost. We model our problem as a deterministic, continuous time optimization problem. Table 2 summarizes the variables, parameters and main notation used in the model in the remainder of the paper.

Table 2: Notation used

Variables:

$X_{N}(t)=$ new products used to satisfy demo requests at time $t$

$X_{R}(t)=$ refurbished products used to satisfy demo requests at time $t$

$X_{M}(t)=$ retired ex-demo products sold in the secondary market at time $t$

$X_{S}(t)=$ retired ex-demo products scrapped at time $t$

$X_{I}(t)=$ inventory of collected demos in the store at time $t$ 
Parameters and other notation:

$t=$ time index

$L=$ demo loan period (residence time with client)

$T=$ time horizon (lifecycle) over which demo requests occur

$d(t)=$ demand for demo products at time $t$

$D(t)=$ cumulative demand for demo products over $[0, t)$

$r(t)=$ ex-demo retirements at time $t$ (to be sold in secondary market or scrapped)

$M(t)=$ cumulative sales revenue in the secondary market over $[L, t)$

$f D(\mathrm{~T})=$ resale potential in the secondary market as a fraction $0 \leq f \leq 1$ of the total demo demand $D(T)$

$N=$ the total number of new products used to satisfy all demo requests

$Q=$ the total number of refurbished ex-demo products sold in the secondary market

$S=$ the total number of ex-demo products scrapped

$c_{N}=$ the new product acquisition cost, per unit

$c_{R}=$ the refurbishment cost, per unit

$c_{S}=$ the scrapping cost, per unit

$c_{I}=$ the inventory holding cost, per unit per unit time

$p_{S}(t)=$ revenue from selling a refurbished ex-demo product in the secondary market at time $t$

Given the above, the model formulation is expressed as:

$$
\begin{gathered}
\text { Minimize } \int_{0}^{L} c_{N} X_{N}(t) d t+\int_{L}^{T} c_{N} X_{N}(t)+c_{R} X_{R}(t) d t+ \\
\int_{L}^{T+L} c_{S} X_{S}(t)+\left(c_{R}-p_{S}(t)\right) X_{M}(t) d t+\int_{L}^{T+L} c_{I} X_{I}(t) d t
\end{gathered}
$$

Subject to:

$$
\begin{aligned}
& X_{N}(t)=d(t) \text { for } t \in[0, L) \\
& X_{N}(t)+X_{R}(t)=d(t) \text { for } t \in[L, T) \\
& X_{I}(t)=0 \text { for } t \in[0, L) \\
& \dot{X}_{I}(t)=d(t-L)-X_{M}(t)-X_{S}(t)-X_{R}(t) \text { for } t \in[L, T) \\
& \dot{X}_{I}(t)=d(t-L)-X_{M}(t)-X_{S}(t) \text { for } t \in[T, T+L) \\
& \int_{L}^{T+L} X_{M}(t) d t \leq f D(T) \\
& X_{N}(t), X_{R}(t), X_{I}(t), X_{M}(t), X_{S}(t) \geq 0 \text { for } t \in[0, T+L)
\end{aligned}
$$


The objective function Eq.(1) minimizes the cost of satisfying demo product requests by either new or refurbished products, the cost of scrapping or salvaging (after refurbishment) ex-demo retirements in the secondary market, and the inventory holding cost at the demo store. Constraints (2) are the demo demand constraints: at any point $t$ in $[0, L)$ all request are satisfied by new products; at any point $t$ in $[L, T)$ requests are satisfied by either new or refurbished products. Constraints (3) are inventory balance constraints for collected demo products at the store. In $[0, L)$ the inventory is zero (no collections available before $L$ ). At any point $t$ in $[L, T)$, the change in inventory is equal to the time-phased demand $d(t-L)$ (i.e., the collections at time $t$ ) minus the products that are refurbished and sold in the market at time $t$, those that are scrapped at time $t$ and those that are refurbished and reused at time $t$. The constraint for $t$ in $[T, T+L)$ is similar, but without the reuse option. Constraint (4) is the secondary market capacity constraint: the total sales in the secondary market is limited to a fraction $f$ of the total demo demand $D(T)$. Constraints (5) are the non-negativity restrictions.

We analyze model (1)-(5) for a concave (or unimodal) demo demand profile and show that a zero inventory policy is optimal. For any concave (or unimodal) profile it is readily observed that the request profile $d(t)$ for $0 \leq t<T$ and time-phased collection profile $d(t-L), L \leq t<T+L$ with $L<T$, intersect at exactly one point $t_{S}$ in time interval $[L, T]$, from where the collections $d(t-L)$ exceed and stay larger than the demand $d(t)$ for the rest of the planning horizon. There are no other intersections between $d(t)$ and $d(t-L)$. This point $t_{S}$ is within in the interval $[L, T)$ when $d(t)$ peaks well before $T$, while $t_{S}=T$ when the peak occurs right at the end or when $d(t)$ is constant When the inventory holding cost $c_{I} \geq 0$ and when revenue in the secondary market erodes, a decision must be made instantly: reuse, sell or scrap. There is no benefit from storing collections temporarily in inventory to fulfil later requests, or delay sales, because costs can only increase and revenue only decrease. If the demand profile $d(t)$ has multiple peaks and troughs (i.e. $d(t)$ and $d(t-L)$ intersect at different points in time), it may be economically attractive to temporarily store excess collections in inventory for later reuse until demand surges. However, this does not happen with a concave (or unimodal) demand profile and it is optimal for the store to operate a zero inventory policy.

With a zero inventory policy, we have $\dot{X}_{I}(t)=X_{I}(t)=0$ for all $t$ and we can remove the inventory cost component from the objective function. In addition, constraints (3) reduce to:

$$
\begin{aligned}
& X_{M}(t)+X_{S}(t)+X_{R}(t)=d(t-L) \text { for } t \in[L, T) \\
& X_{M}(t)+X_{S}(t)=d(t-L) \text { for } t \in[T, T+L)
\end{aligned}
$$

If we now substitute $X_{N}(t)$ and $X_{S}(t)$ in the objective function (1) using Eq.(2) and Eq.(6), our model becomes:

Minimize $\left(c_{N}+c_{S}\right) D(T)-\int_{L}^{T}\left(c_{N}+c_{S}-c_{R}\right) X_{R}(t) d t-\int_{L}^{T+L}\left(p_{S}(t)+c_{S}-c_{R}\right) X_{M}(t) d t$ 
Subject to: (2), (6), (4) and (5).

The first term in Eq.(7) corresponds to the cost of a base demo policy, which involves using a new product for each demo request and scrapping all products after they have been collected. The second term in Eq.(7) shows the potential improvement over the base demo strategy by reusing collected demos. We call $c_{N}+c_{S}-c_{R}$ the reuse saving. The third term in Eq.(7) shows the potential improvement over the base policy by selling retired and refurbished ex-demo products in the secondary market. We call $p_{S}(t)+c_{S}-c_{R}$ the resale saving. When both reuse and resale saving are $\leq 0$ for all $t$, we cannot improve over the base strategy because none of the recovery options are economically attractive. We are interested in the case where both reuse and resale savings are positive and compete against each other. The optimal strategies when either the reuse saving or resale saving are negative can be derived as special, simpler, cases from the results presented in Sections 4 and 5 below.

We note that problem (7), (2), (6), (4) and (5) is a special Separated Continuous Linear Program (SCLP) (see e.g., Pullan, 1996). In the Appendix we show that our problem can be transformed into a Continuous Transportation Problem (CTP), for which strong duality results exist (Anderson and Philpott, 1984) that resemble the well-known strong duality results for static Transportation Problems. We use these conditions to prove the optimality of the different solution structures that we derive in the next two sections. The solution to our problem changes at discrete points in time. Our analysis in the following sections focuses on identifying (using cost economic analysis) those moments in the planning horizon where these switches occur.

First, we derive two signals that drive the optimal solution to our problem. Consider a collected demo product and analyze if it is more profitable to reuse this product for a next request, or better to satisfy the next request by a new product and sell the collected ex-demo product in the secondary market. All constraints, except constraint (4), in our model are equality constraints. We distinguish two scenarios for constraint (4): a non-binding secondary market capacity constraint or a binding secondary market constraint.

When there is no restriction on the sales potential in the secondary market (constraint (4) is not binding), in the reuse option we satisfy the new request at time $t$ by refurbishing and reusing the available collected ex-demo product $\left(\cos t c_{R}\right)$. After the demo loan period $L$, this product is collected, refurbished $\left(\operatorname{cost} c_{R}\right)$ and sold in the secondary market, at price $p_{S}(t+L)$. The total cost for the reuse option is $2 c_{R}-p_{S}(t+L)$. In the no-reuse option, we use a new product to satisfy the request at time $t$ $\left(\operatorname{cost} c_{N}\right.$ ). Both the available collected ex-demo product at time $t$ and the new product collected at time $t+L$ will be refurbished and sold in the secondary market, at prices $p_{S}(t)$ and $p_{S}(t+L)$. The total cost for the no-reuse scenario is $c_{N}+2 c_{R}-p_{S}(t)-p_{S}(t+L)$. The reuse scenario is preferred over the no-reuse scenario as long as $p_{S}(t) \leq c_{N}$. With non-increasing $p_{S}(t)$, the point in time when $p_{S}(t)$ drops below $c_{N}$ is a signal to start reuse. We refer to the case where $p_{S}(t) \geq c_{N}$ for all $t$ as the case where selling in the 
secondary market is 'profitable'. When $p_{S}(t)$ drops below $c_{N}$ selling in the secondary market becomes unprofitable.

When the secondary market constraint becomes binding, we assume that we can still sell one more product in the secondary market. The cost for the reuse option is as before $2 c_{R}-p_{S}(t+L)$. In the no-reuse scenario, the only difference is that a new product that was used to satisfy the demo request at time $t$, will be scrapped (cost $c_{S}$ ) after collection at time $t+L$. The total cost for the no-reuse option is then $c_{N}+c_{R}+c_{S}-p_{S}(t)$. Reuse is preferred when $p_{S}(t)-p_{S}(t+L)=\Delta p_{S}(t) \leq c_{N}+c_{S}-c_{R}$. A large drop in the sales revenue between selling now and later (i.e. larger than the reuse saving) is an indicator to retire and sell ex-demo equipment in the secondary market early and postpone reuse until later in the lifecycle (even if this involves scrapping the later retirements). We denote the case where $\Delta p_{S}(t) \leq c_{N}$ $+c_{S}-c_{R}$ as the low erosion case, and the case where $\Delta p_{S}(t)>c_{N}+c_{S}-c_{R}$ as the high value erosion case.

Using these two indicators, we consider four scenarios for further analysis:

Case 1: $p_{S}(t) \geq c_{N}$ and $\Delta p_{S}(t) \leq c_{N}+c_{S}-c_{R}$ : selling in the secondary market is 'profitable' and there is low value erosion

Case 2: $p_{S}(t)$ drops below $c_{N}$ and $\Delta p_{S}(t) \leq c_{N}+c_{S}-c_{R}$ : selling in the secondary market becomes unprofitable at some time and there is low value erosion

Case 3: $p_{S}(t) \geq c_{N}$ and $\Delta p_{S}(t)>c_{N}+c_{S}-c_{R}$ : selling in the secondary market is 'profitable' and there is high value erosion

Case 4: $p_{S}(t)$ drops below $c_{N}$ and $\Delta p_{S}(t)>c_{N}+c_{S}-c_{R}$ : selling in the secondary market becomes unprofitable at some time and there is high value erosion

We derive the optimal demo strategy for the low value erosion cases in Section 4. The optimal strategies for the high value erosion cases are presented in Section 5. In the Appendix we prove the optimality of the proposed solution structures using strong duality and reduced cost results for the CTP.

\section{Derivation of the optimal product demo policies with low value erosion}

\subsection{Selling in the secondary market is always profitable $\left(p_{S}(t) \geq c_{N}\right)$}

When $p_{S}(t) \geq c_{N}$ it is profitable to sell ex-demo products in the secondary market and we should exploit this option to its full potential. This condition also implies that the resale saving $p_{S}(t)+c_{S}-c_{R}$ is larger than the reuse saving $c_{N}+c_{S}-c_{R}$. Resale is the preferred value recovery option at any time. The optimal amount of products sold in the secondary market is equal to the secondary market potential: $Q$ $=f D(T)$. While there is an incentive to sell as early as possible $\left(p_{S}(t)\right.$ declines over time), there is no incentive to sell a collected demo product early in the lifecycle if this product could still be reused to satisfy a future demo request and if this would result in more scrapping (low value erosion). The total number of new products used to satisfy all demo requests over the lifecycle $T$ is $N=\operatorname{Max}\left\{N_{\min }(L)\right.$; $f D(T)\} . N_{\min }(L)$ is the minimum number of new products required to satisfy all demo requests and can 
be calculated (Eq.(8)) from the demo request profile $d(t)$ and searching for the maximum number of requests over any time window of length $L$ in the planning horizon $T$ :

$$
N_{\text {min }}(L)=\operatorname{Max}_{0 \leq t \leq T-L}\left\{\int_{t}^{t+L} d(t) d t\right\} .
$$

We consider two situations.

Case 4.1.(a): $N_{\min }(L)>f D(T)$

The number of new products used to satisfy all requests is $N=N_{\min }(L)$. New products are used for all demo requests before $L$ and for the requests in excess of available collected demos between $L$ and $t_{s}$ (i.e., $d(t)-d(t-L))$. $t_{S}$ denotes the time when the collections exceed and remain larger than the requests. Reuse starts at time $L$ : between $L$ and $t_{s}$ all collections are reused; between $t_{s}$ and $T$, only the part of the collections required to satisfy new requests are reused (i.e., $d(t-L)-d(t))$. The demo retirement profile (i.e., ex demos to be scrapped or sold in the secondary market) is $r(t)=X_{M}(t)+X_{S}(t)=\operatorname{Max}\{0 ; d(t-L)$ $d(t)\}$ for $L \leq t<T+L$. Only the earliest retirements are sold in the secondary market (up to the market constraint $f D(T)$ ); later retirements are scrapped. Let us define $t_{f}$ as the market saturation time, i.e., such that:

$$
\int_{L}^{t_{f}} \operatorname{Max}\{0 ; d(t-L)-d(t)\} d t=f D(T)
$$

The secondary market revenue can then be calculated as $M\left(t_{f}\right)=\int_{L}^{t_{f}} p_{S}(t) r(t) d t$, and the total amount of scrapped ex-demo products is $S=\operatorname{Max}\left\{0, N_{\min }(L)-f D(T)\right\}$. The policy is illustrated in the left-hand diagrams, Figure 5(a): the diagram on the top shows a triangular requests $d(t)$ and collection $d(t-L)$ profile, the requests that are satisfied by new products $\left(X_{N}(t)\right.$ variable) and the collected demos that are scrapped after market saturation time $t_{f}$. $\left(X_{S}(t)\right.$ variable). In this example $L=5, T=20, t_{s}=12.5 ; t_{f}=$ 22.5. The area where request and collection profile overlap corresponds to the collections that are reused. The reuses $X_{R}(t)$ are also shown in the diagram at the bottom as well as retired ex-demos sold in the market $X_{M}(t)$. 
Policy 4.1.a: requests, collections, $\mathrm{X}_{N}(\mathrm{t})$ and $\mathrm{X}_{\mathrm{S}}(\mathrm{t})$

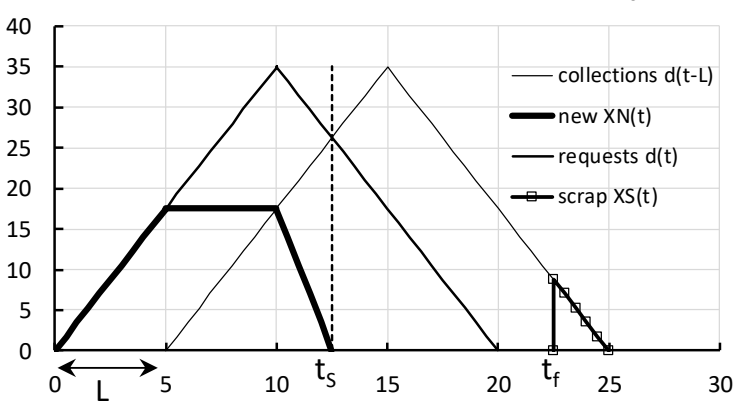

Policy 4.1.a: collections, $\mathrm{X}_{\mathrm{R}}(\mathrm{t})$ and $\mathrm{X}_{\mathrm{M}}(\mathrm{t})$

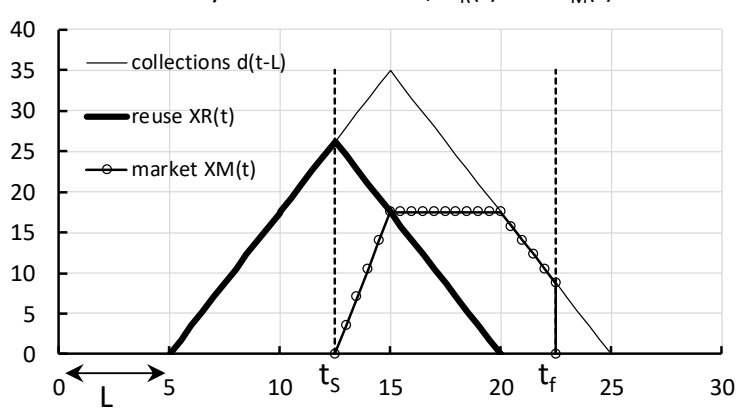

Policy 4.1.b: requests, collections and $\mathrm{X}_{N}(\mathrm{t})$

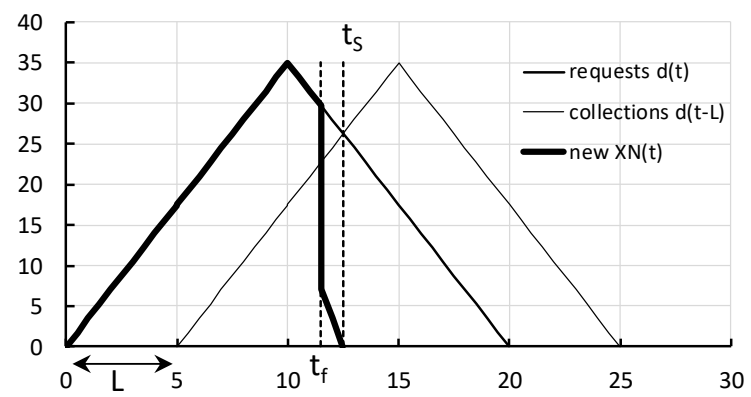

Policy 4.1.b: collections, $X_{R}(t)$ and $X_{M}(t)$

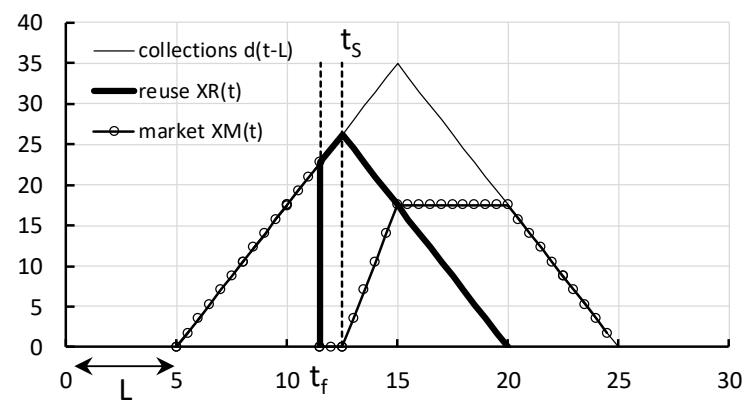

Figure 5: Optimal demo policy when selling in the secondary market is profitable and value erosion is low for (a) $N_{\min }(L)>f D(T)$ (left) and (b) $N_{\min }(L) \leq f D(T)$ (right).

Case 4.1.(b): $N_{\min }(L) \leq f D(T)$

When $N_{\min }(L) \leq f D(T)$, it is optimal to use more than $N_{\min }(L)$ new products to satisfy all demo requests. The extra amount of new products $E\left(t_{f}\right)$ is determined by a market saturation time $t_{f}$. In this situation, we apply the $N_{\min }(L)$ new products ordering strategy (like in Case 4.1.(a)), but with no reuse of the collections in period $\left[L, t_{f}\right)$. The earliest collections in $\left[L, t_{f}\right)$ are retired and sold in the secondary market, and $E\left(t_{f}\right)$ additional new products are required to satisfy demo requests in $\left[L, t_{f}\right)$. $E\left(t_{f}\right)$ is calculated through Eq.(10):

$$
E\left(t_{f}\right)=\int_{L}^{t_{f}} \operatorname{Min}\{d(t) ; d(t-L)\} d t \text { and such that } f D(T)=N_{\min }(L)+E\left(t_{f}\right) .
$$

The retirement profile is $r(t)=d(t-L)$ for $L \leq t<t_{f}$ and $r(t)=\operatorname{Max}\{0 ; d(t-L)-d(t)\}$ for $t_{f} \leq t<T+L$. All retired ex-demo equipment is sold in the secondary market and there is no scrapping. This policy is illustrated in the right-hand diagrams, Figure 5(b). We show the same characteristics as in Figure 5(a) and the diagram is for $t_{f}=11.5$. We note that, in the diagram on the top, the new products $X_{N}(t)$ follow the demo request $d(t)$ profile until $t_{f}$. In the diagram at the bottom, collections between $L$ and $t_{f}$ are sold in the market. Reuse $\left(X_{R}(t)\right)$ starts at $t_{f}$ but the collections are not sufficient to satisfy all requests between $t_{f}$ and $t_{s}$. The $X_{N}(t)$ curve shows indeed that, in this example, new products are used to satisfy 
some requests between $t_{f}$ and $t_{s}$. After $t_{s}$, all excess collections (i.e., $\left.d(t-L)-d(t)\right)$ are retired and sold in the secondary market. There is no scrapping in this scenario.

\subsection{Selling in the secondary market becomes unprofitable at $t^{*}\left(p_{S}(t)<c_{N}\right)$}

As long as the resale saving $p_{S}(t)+c_{S}-c_{R} \geq 0$, some value is recovered from selling in the secondary market. Because we assume low erosion $\left(\Delta p_{S}(t) \leq c_{N}+c_{S}-c_{R}\right)$ and $p_{S}(t)$ is higher than $c_{N}$ (for $\left.t<t^{*}\right)$, the resale saving $p_{S}(t)+c_{S}-c_{R}$ is always positive (i.e., when $c_{R} \geq c_{S}$, which is a reasonable assumption). At $t^{*}$, the preferred value recovery option switches to reuse. It is straightforward to modify the optimal strategies from Section 4.1 to the case when resale becomes unprofitable at time $t^{*}$. We consider the same two scenarios:

Case 4.2.(a): $N_{\min }(L)>f D(T)$ :

When $N_{\min }(L) \geq f D(T)$, we need more new products to satisfy all demo requests than we could possibly sell in the secondary market. Whether $p_{S}(t)$ drops below $c_{N}$ will not affect the optimal policy and the best strategy is as explained in Section 4.1 (Figure 5(a)) with $N=N_{\min }(L)$ and $Q=f D(T)$.

Case 4.2.(b): $N_{\min }(L) \leq f D(T)$ :

When $N_{\min }(L) \leq f D(T)$, it is important to know if selling in the secondary market becomes unprofitable early in the lifecycle $\left(t^{*}<L\right)$, or later $\left(t^{*} \geq L\right)$. In the case where $t^{*}<L$, every retired ex-demo product sold in the secondary market yields a loss. We want to exploit the reuse option as much as possible and use only $N=N_{\min }(L)$ new products to satisfy all requests (the reuse saving is larger than the resale saving). All $N_{\min }(L)$ products will be sold in the secondary market after collection and when they are not needed anymore. This policy can also be visualized from Figure 5.1.(a), with the only difference that there is no $t_{f}$ and selling in the secondary market continues until $T+L$. The secondary market capacity constraint is not binding and there is no scrapping.

In the case where $t^{*} \geq L$, the optimal policy is to use more than $N_{\min }(L)$ new products to satisfy all demo product requests. As in Section 4.1, we can determine a market saturation time $t_{f}$ through Eq.(10). When $t_{f}<t^{*}$, i.e., we use $N=f D(T)$ new products in total to satisfy all demo requests and only start reuse after $t_{f}$, which is the same policy as in case 4.1.(b), Figure 5(b). When $t_{f}>t^{*}$, however, it is better to start reuse just after time $t^{*}$ and we do not saturate the entire secondary market. The optimal strategy is therefore to postpone reuse until $\operatorname{Min}\left\{t_{f}, t^{*}\right\}$.

\subsection{Managerial insights}

The analysis of the low erosion case reveals that several problem parameters are key in order to derive and formulate an optimal demo strategy. Time related and/or time varying characteristics are of particular importance to capture the dynamic nature of the policies and to identify the exact moments in time when policy switches occur. The length of the demo loan period $L$ relative to the length of the 
product's lifecycle $T$ are two clear problem characteristics that impact the value recovery options. When $L$ is long relative to $T$ there is less opportunity for reuse and more new products are required to satisfy all demo requests. With eroding secondary market sales prices $p_{s}(t)$, less revenue is recovered the longer demo products reside at the client sites.

The demo loan period $L$ can have a strong impact on $N_{\min }(L)$ - the minimum number of new products required to satisfy all requests (see Eq.(8)). $N_{\min }(L)$ is a critical policy and cost driver in this problem environment and it is important to understand its sensitivity to changes in $L$ and in the demand profile $d(t) . N_{\min }(L)$ is larger for demo request profiles with a more pronounced peak. We illustrate this in Figure 6, which shows four request profiles for $0 \leq t<20$ and the sensitivity of $N_{\min }(L)$ to changes in $L$ : profile 1: constant demand $(d(t)=50)$; profile 2: concave decreasing $\left(d(t)=75-\frac{3 t^{2}}{16}\right)$; profile 3 : decreasing right triangular $(d(t)=100-5 t)$; and profile 4: convex decreasing $\left(d(t)=\frac{3(t-20)^{2}}{8}\right)$. Each profile has a total demand $D(20)=1000$. Profile 4 has the most pronounced peak.
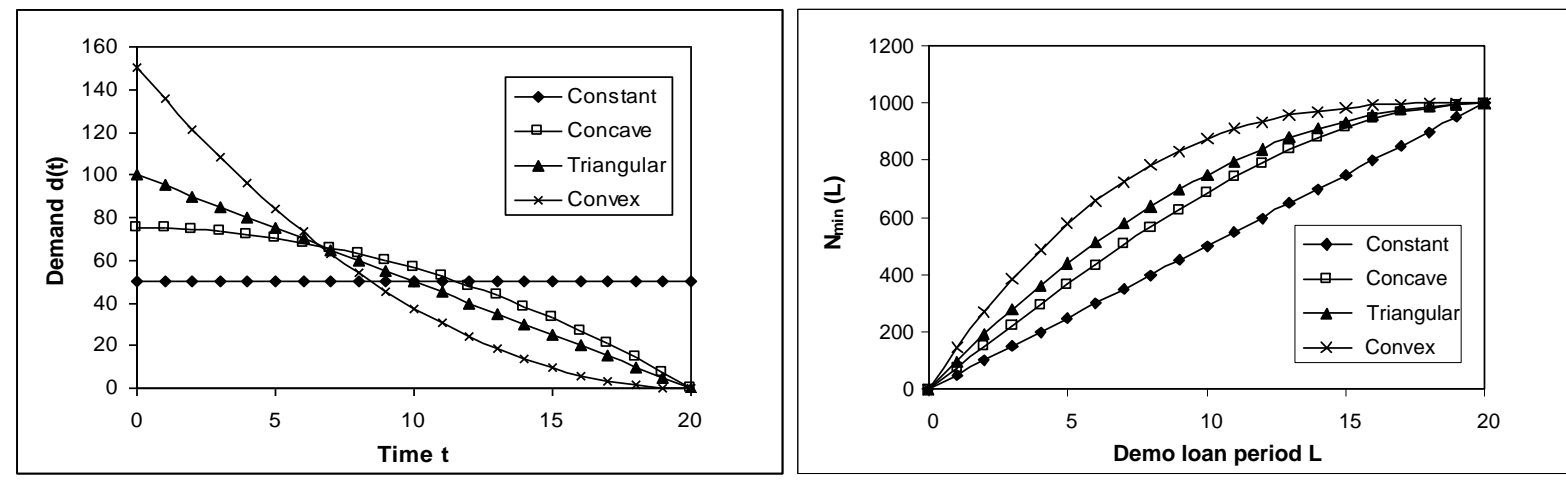

Figure 6: $N_{\min }(L)$ as a function of $L$ for different profiles

From Figure 6 it is readily observed that (keeping $L$ constant) $N_{\min }(L)$ is larger for profiles with a more pronounced peak, and when $L$ is small, $N_{\min }(L)$ is more sensitive to changes in $L$ for profiles with a higher peak. Conversely, when $L$ is large, $N_{\min }(L)$ becomes less sensitive to changes in $L$ for profiles with a higher peak. Demo managers need to be aware of the demo request profile and its impact on the subsequent reuse and recovery operations. Profiles that are as level possess the greatest value recovery potential when reuse is the only option to avoid value loss.

The demo request profile $d(t)$ and loan period $L$ also define $t_{S}$ - the point in time when collections exceed and stay larger than the requests. As shown in Sections 4.1 and 4.2, this is an important policy switch point because excess collections must find a final destination, either through scrapping or by sales in the secondary market. The shape of the request profile impacts on the position of $t_{S}$ : it occurs somewhere within active lifecycle $[0, T)$ for profiles that peak before $T$, or at time $T$ for constant demand profiles and profiles with a peak demand at time $T$.

The relations between revenue $p_{S}(t)$ and cost $c_{N}$ and between the sales potential in the secondary market $f D(T)$ and $N_{\min }(L)$ are the two another policy defining factors. These define the possible switching point $t^{*}$, where resale in the secondary market becomes unattractive, and a market saturation 
time $t_{f}$. Each of these factors interact and may influence each other. It is therefore not possible to define an optimal demo strategy based just on partial information (e.g., simply comparing $p_{s}(t)$ and $c_{N}$ while ignoring $f \mathrm{D}(T)$ and $N_{\min }(L)$ ). All characteristics have to be considered as well as the positions of different switching points $\left(t_{S}, t^{*}, t_{f}\right)$ in relation to $L$ and $T$.

\section{Derivation of the optimal demo policies with high value erosion}

\subsection{Selling in the secondary market is always profitable $\left(p_{S}(t) \geq c_{N}\right)$}

As in Section 4.1, when $p_{S}(t) \geq c_{N}$, it is profitable to sell in the secondary market and this recovery option should be exploited to its full potential. The total number of ex-demo products sold in the secondary market is $Q=f D(T)$. In addition, because the revenue profile $p_{S}(t)$ exhibits large price drops, it may be better to sell collected ex-demo products early in the lifecycle and use new products to satisfy subsequent demo requests, even when these products have to be scrapped after collection. In other words, it can be optimal to use more than $\operatorname{Max}\left\{N_{\min }(L) ; f D(T)\right\}$ new products to satisfy all demo requests. We analyze the same two scenarios as before:

Case 5.1.(a): $N_{\min }(L)>f D(T)$

Let the market saturation time $t_{f}$ be defined as in Case 4.1.(a), Eq.(9) and let $\Delta t$ and $\Delta t_{f}$ denote small time epochs. When the difference in revenue between selling at time $L+\Delta t$ and $t_{f}-\Delta t_{f}$ is bigger than the reuse saving, i.e., $p_{S}(L+\Delta t)-p_{S}\left(t_{f}-\Delta t_{f}\right)>c_{N}+c_{S}-c_{R}$, then the latest sold ex-demo products in Figure 5(a).1 (time period $\left[t_{f}-\Delta t_{f}, t_{f}\right)$ ) will be scrapped, whereas the earliest collections (time period $[L, L+\Delta t))$ will be sold in the secondary market (instead of being reused). To satisfy the demo requests in time period $[L, L+\Delta t)$, new products are used. The key question is therefore to determine the largest possible value for $\Delta t$ (and the corresponding value for $\left.\Delta t_{f}\right)$ such that $p_{S}(L+\Delta t)-p_{S}\left(t_{f}-\Delta t_{f}\right) \geq c_{N}+c_{S}-$ $c_{R}$ and,

$$
\int_{L}^{L+\Delta t} \operatorname{Min}\{d(t) ; d(t-L)\} d t=\int_{t_{f}-\Delta t_{f}}^{t_{f}} \operatorname{Max}\{0 ; d(t-L)-d(t)\} d t
$$

The left hand side in Eq. (11) comprises of the collections in $[L, L+\Delta t$ ) that can possibly be reused; the right hand side in Eq. (11) are excess collections in $\left[t_{f}-\Delta t_{f}, t_{f}\right)$ that can only be sold in the market or be scrapped. We call the left integral in Eq.(11), $E(L+\Delta t)$, that is, the extra number of new products (in addition to $\left.N_{\min }(L)\right)$ that will be used to satisfy all demo requests.

A convenient way to find the values for $\Delta t$ (and $\Delta t_{f}$ ) is to consider the function $\Delta p_{S}(t)=p_{S}(t)-$ $p_{S}(t+L)$, which compares the sales revenues at times $t$ and $t+L$. If $\Delta p_{S}(t)$ is smaller than the reuse saving $c_{N}+c_{S}-c_{R}$, value erosion is low (see Section 4). Otherwise, when $\Delta p_{S}(t)>c_{N}+c_{S}-c_{R}$ for some values of $t$, we look for the largest value of $t(L \leq t \leq T)$ for which $\Delta p_{S}(t)>c_{N}+c_{S}-c_{R}$. Let us denote this $t$ - 
value by $t_{R}$. We can set $t_{R}=L+\Delta t$ as the upper limit in the left integral in Eq.(11), and find the corresponding $\Delta t_{f}$ value from Eq.(11). This approach ignores the secondary market capacity constraint. We will never use more extra new products than we could possibly sell in the market (i.e., $f D(T)$ ). The limiting value for this constraint $E(L+\Delta t)=f D(T)$ thus defines another candidate upper limit $t_{C}$ for $L+\Delta t$. Therefore, we can set $L+\Delta t=\operatorname{Min}\left\{t_{R}, t_{C}\right\}$ and require $p_{S}(L+\Delta t)-p_{S}\left(t_{f}-\Delta t_{f}\right) \geq c_{N}+c_{S}-c_{R}$.

The optimal demo retirement profile is $r(t)=d(t-L)$ for $L \leq t<L+\Delta t$ and $r(t)=\operatorname{Max}\{0 ; d(t-L)$ $d(t)\}$ for $L+\Delta t \leq t<T+L$. The secondary market revenue equals $M\left(t_{f}-\Delta t_{f}\right)=\int_{L}^{t_{f}-\Delta t_{f}} p_{S}(t) r(t) d t$. All retirements after $t_{f}-\Delta t_{f}$ are scrapped.

Figure 7(a) illustrates this policy. We show the same problem characteristics as in Figure 5(a). Compared with the top diagram in Figure 5(a), we continue to use extra new products to satisfy demo requests for time $\Delta t$. Because the market capacity is limited, the same quantity of products has to be scrapped, as indicated by the earlier start of the $X_{S}(t)$ curve. In the bottom part of the diagram we observe that reuse, $X_{R}(t)$ is postponed by $\Delta t$ and that the secondary market sales, $X_{S}(t)$ follow the collections $d(t-L)$ from $L$ to $L+\Delta t$, but also finish $\Delta t_{f}$ earlier.
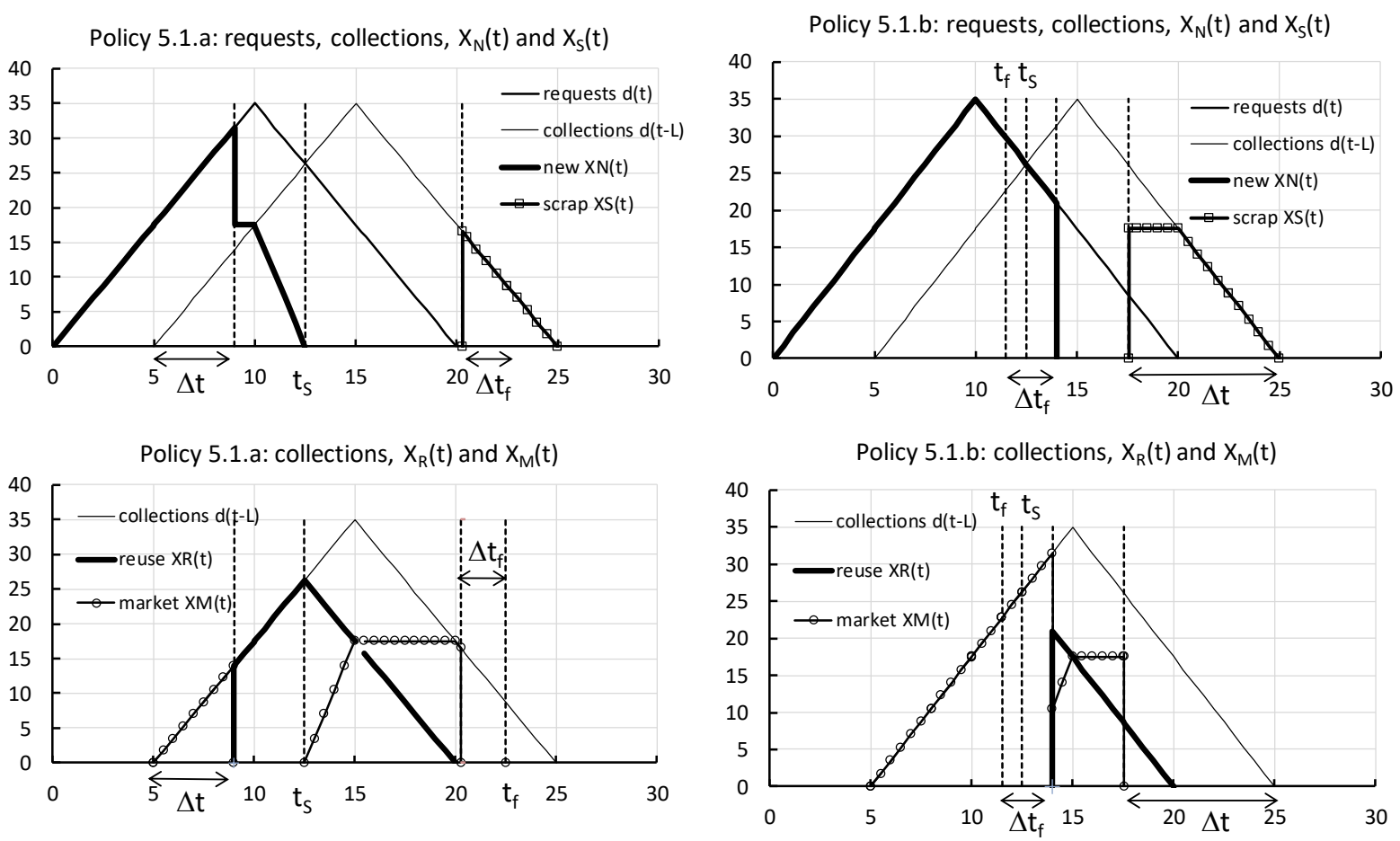

Figure 7: Optimal demo policy when selling in the secondary market is profitable and value erosion is high for (a) $N_{\min }(L)>f D(T)$ (left) and (b) $N_{\min }(L) \leq f D(T)$ (right).

Case 5.1.(b): $N_{\min }(L) \leq f D(T)$

Similar reasoning may be used for the case where $N_{\min }(L) \leq f D(T)$. The optimal strategy is to use more than $f D(T)=N_{\min }(L)+E\left(t_{f}\right)$ new products to satisfy all demo requests (Section 4.1), yet we can sell only $Q=f D(T)$ ex-demo products in the secondary market. In order to determine the extra new products 
required, we find the largest value for $\Delta t_{f}$ (and corresponding value for $\left.\Delta t\right)$ such that $p_{S}\left(t_{f}+\Delta t_{f}\right)-p_{S}(T+L-$ $\Delta t) \geq c_{N}+c_{S}-c_{R}$ and

$$
\int_{t_{f}}^{t_{f}+\Delta t_{f}} \operatorname{Min}\{d(t) ; d(t-L)\} d t=\int_{T+L-\Delta t}^{T+L} \operatorname{Max}\{0 ; d(t-L)-d(t)\} d t
$$

The left hand side in Eq.(12) comprises of collections in $\left[t_{f}, t_{f}+\Delta t_{f}\right.$ ) that can possibly be reused. The right hand side in Eq. (12) are collections in excess of the demo product demand $d(t)$ in $[T+L-\Delta t, T+L)$ that can only be sold in the market or be scrapped. We denote the left integral in Eq.(12) by $E\left(t_{f}+\Delta t_{f}\right)$. As before, the values for $\Delta t_{f}$ and $\Delta t$ can be found from the function $\Delta p_{S}(t)=p_{S}(t)-p_{S}(t+L)$. One candidate point for $t_{f}+\Delta t_{f}$, is the largest $t$-value (called $t_{R}$ ) for which $\Delta p_{S}(t)>c_{N}+c_{S}-c_{R}$. The other candidate point $t_{C}$ is determined by the limit value of a 'reduced' secondary market capacity constraint $E\left(t_{f}+\Delta t_{f}\right)=f D(t)-E\left(t_{f}\right)=N_{\min }(L)$. Note that the market capacity must be reduced by the early sales $E\left(t_{f}\right)$, which generate the most revenue. We can then set $t_{f}+\Delta t_{f}=\operatorname{Min}\left\{t_{R}, t_{C}\right\}$, and the value for $\Delta t$ can be calculated from Eq.(12).

The optimal ex-demo retirement profile is $r(t)=X_{M}(t)+X_{S}(t)=d(t-L)$ for $L \leq t<t_{f}+\Delta t_{f}$ and $r(t)$ $=\operatorname{Max}\{0 ; d(t-L)-d(t)\}$ for $t_{f}+\Delta t_{f} \leq t<T+L$. Ex-demo products retiring after $T+L-\Delta t$ are scrapped. The

revenue in the secondary market is $M(T+L-\Delta t)=\int_{L}^{T+L-\Delta t} p_{S}(t) r(t) d t$. This policy is illustrated in Figure 7(b). Comparing the top diagrams in Figures 5(b) and 7(b), it is evident that we continue to use extra new products to satisfy demo requests for time $\Delta t_{f}$. While there was no scrapping in Figure 5(b), an extra amount of products $E\left(t_{f}+\Delta t_{f}\right)$ are scrapped in Policy 5.1.(b), as shown by the $X_{S}(t)$ curve starting at $T+L-\Delta t$. In the bottom part of the diagram we also observe that reuse, $X_{R}(t)$ is postponed until $t_{f}+\Delta t_{f}$ and that the secondary market sales, $X_{S}(t)$ follow the collections $d(t-L)$ from $L$ until $t_{f}+\Delta t_{f}$, but also finish $\Delta t$ earlier.

\subsection{Selling in the secondary market becomes unprofitable at $t^{*}\left(p_{S}(t)<c_{N}\right)$}

As in Section $4.2 p_{S}(t)$ dropping below $c_{N}$ at time $t^{*}$ is a signal to start reuse. We can modify the optimal policies in Section 5.1 in a similar fashion as we modified the optimal strategies in Section 4.1.

Case 5.2.(a): $N_{\min }(L)>f D(T)$

When $t^{*}<L$, we already need more new products to satisfy all demo demands than we could possibly sell in the secondary market. When selling in the secondary market becomes unprofitable very early in the lifecycle before any collections are available $\left(t^{*}<L\right)$, the reuse recovery option is always preferred. The optimal strategy is to use only $N=N_{\min }(L)$ new products to satisfy all demo requests. As long as 
the resale saving $p_{S}(t)+c_{N}-c_{R}$ is positive, we will sell retired ex-demo products in the secondary market until the market is saturated. In case the resale saving becomes negative at some time $t_{N}$ before the market saturation time $t_{f}$ (see Figure 5(a)), secondary market sales will be halted and all retirements after $t_{N}$ will be scrapped.

When $t^{*} \geq L, \Delta t$ can be determined as explained in Case 5.1.(a). Note that in Section 5.1 reuse is postponed until time $L+\Delta t$. Because the reuse option is preferred over the resale option at time $t^{*}$, we can modify the Section 5.1 policy so as to start reuse at time $\operatorname{Min}\left\{L+\Delta t ; t^{*}\right\}$. The optimal number of new products to satisfy all demo requests is $N=N_{\min }(L)+E\left(\operatorname{Min}\left\{L+\Delta t ; t^{*}\right\}\right)$. With respect to the resale revenue in the secondary market, we halt sales in the secondary market as soon as the resale saving becomes negative or when the market is saturated, whichever occurs earliest.

Case 5.2.(a): $N_{\min }(L) \leq f D(T)$

When $t^{*}<L$, the optimal strategy is to use $N=N_{\min }(L)$ new products to satisfy all demo requests. We stop selling in the secondary market as soon as the resale saving becomes negative.

When $t^{*} \geq L, \Delta t_{f}$ is determined as in Case 5.1.(b). Because the reuse option is preferred over the resale option at time $t^{*}$, we can modify the Section 5.1 policy so as to start reuse at time $\operatorname{Min}\left\{t_{f}+\Delta t_{f}\right.$, $\left.t^{*}\right\}$. The optimal number of new products used to satisfy all demo requests is given by $N=$ $N_{\min }(L)+E\left(\operatorname{Min}\left\{t_{f}+\Delta t_{f}, t^{*}\right\}\right)$. When $L \leq t^{*}<t_{f}$, reuse starts right at time $t^{*}$, i.e. earlier than $t_{f}$ (see Figure 7(b)); when $L \leq t_{f}+\Delta t_{f} \leq t^{*}<T$, reuse is as in Figure 7(b); when $L \leq t_{f} \leq t^{*}<t_{f}+\Delta t_{f}$ reuse starts at $t^{*}$ between $t_{f}$ and $t_{f}+\Delta t_{f}$. With respect to the resale revenue in the secondary market, sales in the secondary market are halted as soon as the resale saving becomes negative (time $t_{N}$ ) or when the market is saturated, whichever occurs earliest.

\subsection{Managerial insights}

High value erosion may occur when the drop in resale revenue over the demo loan period $\Delta p_{S}(t)=p_{S}(t)$ $-p_{S}(t+L)$ is larger than the reuse saving $c_{N}+c_{S}-c_{R}$ and when the secondary market capacity constraint is binding. From an economic perspective it is better to salvage available collections in the secondary market and to keep satisfying demo requests with new products. Reuse is postponed until later, i.e., until $\Delta p_{S}(t)$ drops below $c_{N}+c_{S}-c_{R}$, or until the early sold collections have saturated the secondary market. The high erosion case is less intuitive and more complex than the low erosion case. The key piece of information needed to establish the presence of high value erosion is the $\Delta p_{S}(t)$-function, which is defined or relevant in time interval $[L, T)$. For constant value erosion (e.g., linear price decay), $\Delta p_{s}(t)$ is constant. For more complex price decay (e.g., exponential or piecewise linear decay with discrete drops in sales price) $\Delta p_{S}(t)$ is dynamic and changes over time. The decision of whether high erosion applies may thus change during the lifecycle. This makes sense because product returns early in the lifecycle (e.g., before the price drops in Figure 2) may be prone to high erosion whereas product returns 
near the end of the lifecycle (e.g. after the price drops in Figure 2) may have lost most of their value or may have missed the high revenue time window in the secondary market. Further delays for these last returns may have only limited financial impact so that low erosion applies. $\Delta p_{S}(t)$ also depends on the demo loan period $L$ : the longer $L$, the higher $\Delta p_{S}(t)$ for the same value of $t$. However, $\Delta p_{S}(t)$ is defined over a longer time interval $[L, T)$ when $L$ is lower. Short or medium demonstration times $L$ could make products at some time during the lifecycle prone to high value erosion, whereas (very) long demonstration times could mean that the products have lost most of their resale value after demonstration, and should be controlled by a low erosion strategy.

The high erosion case introduces additional, potential policy switching points $\left(t_{R}, t_{C}\right.$, and $\left.t_{N}\right)$. The point $t_{N}$ is the time when resale revenue becomes negative and sales in the secondary market should stop. The point $t_{R}$ is the largest time for which $\Delta p_{S}(t)>c_{N}+c_{S}-c_{R}$ and $t_{C}$ is a market saturation time for selling early collections. The minimum of $t_{R}$ and $t_{C}$ define a reuse postponement time $\left(\Delta t\right.$ or $\left.\Delta t_{f}\right)$.

\section{Case example}

We now apply our generic analysis above to develop optimal demo policies for case company X noted in Section 2. Figure 8 (left) shows the demo request profile of processor 1 over its 20 months lifecycle. In total 298 requests were received with an average demo loan period around 4.5 months (132 days see Table 1). We base our calculations on an approximation by an isosceles triangular profile $(d(t)=3.5 t$ for $t<10$ and $d(t)=35-3.5(t-10)$ for $10 \leq t \leq 20)$. This approximation provides a close fit for the peak in the real demo requests profile but overestimates the total demo demand (350 units in the approximation vs. 298 in real life). Figure 8 (right) depicts the price erosion of the processor in the secondary market: $p_{S}(t)=\$ 6000-\$ 100 t$ when $t<11$ and $p_{S}(t)=\$ 2900-\$ 100(t-11)$ when $t \geq 11$. The price drop of $\$ 2000$ at $t=11$ corresponds to the introduction of a new generation processor. We assume $c_{N}=\$ 2400, c_{S}=\$ 0$ and $c_{R}=\$ 0$ and that the secondary market sales capacity is limited to 180 units. Figure 8 (right) also shows the $\Delta p_{S}(L)$ curves for four demo loan period values: $L=2.5$ months, $L=4.5$ months, $L=6.5$ months and $L=12$ months.
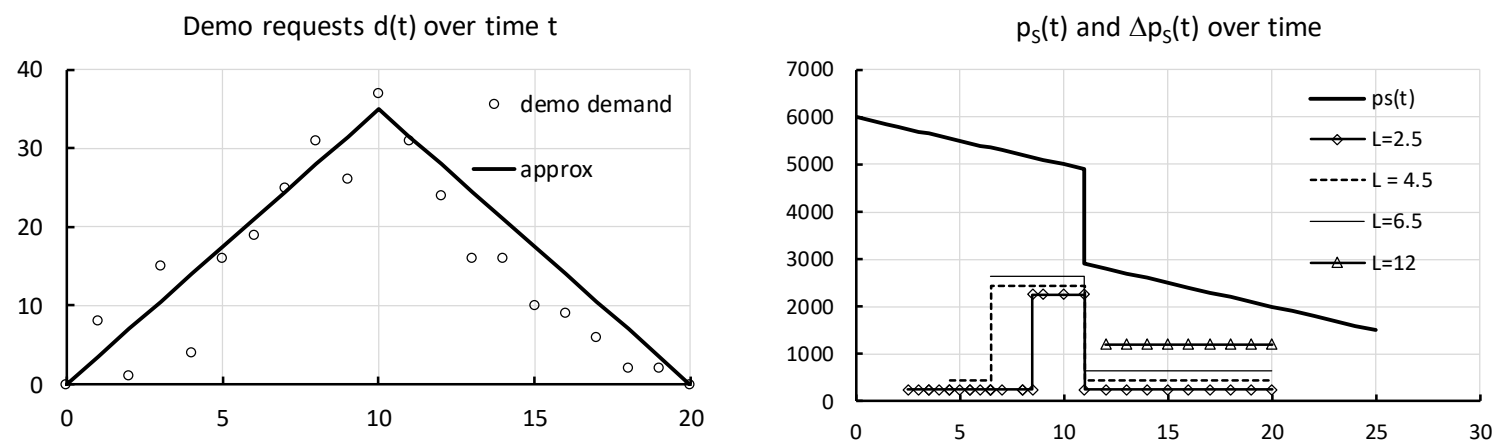

Figure 8: Demo request profile and price erosion of processor 1.

The $\Delta p_{S}(t)$ curves for $L<11$ show a step in time interval [11- $\left.L, 11\right)$, i.e., before the price drop at $t=11$, where they reach a value of $\$ 2000+\$ 100^{*} L$, i.e., the revenue drop at $t=11$ plus the constant erosion 
rate during the loan period $L$. Outside the interval [11- $L, 11)$, the $\Delta p_{S}(t)$ curve show a constant level of $\$ 100^{*} L$. The reuse saving $c_{N}+c_{S}-c_{R}=\$ 2400$. The situations where $L=2.5$ months and $L=12$ months are low erosion cases because $\Delta p_{S}(t)$ only reaches a maximum level of $\$ 2250$ ( $L=2.5$ months) or $\$ 1200$ ( $L=12$ months); the situations where $L=4.5$ months or $L=6.5$ months are high erosions cases $\left(\Delta p_{S}(t)\right.$ reaches maximum values of $\$ 2450$ and $\$ 2650$, which is higher than the $\$ 2400$ reuse saving). Before we discuss each of the four cases in more detail, we present a summary policy map in Figure 9. The diagram shows the optimal demo policy as a function of the demo loan period $L$ and the secondary market sales potential $f$ for the triangular demand profile and for the given price erosion profile $p_{S}(t)$.

\section{Policy regions as a function of $L$ (horizontal} axis) and $f$ (vertical axis)

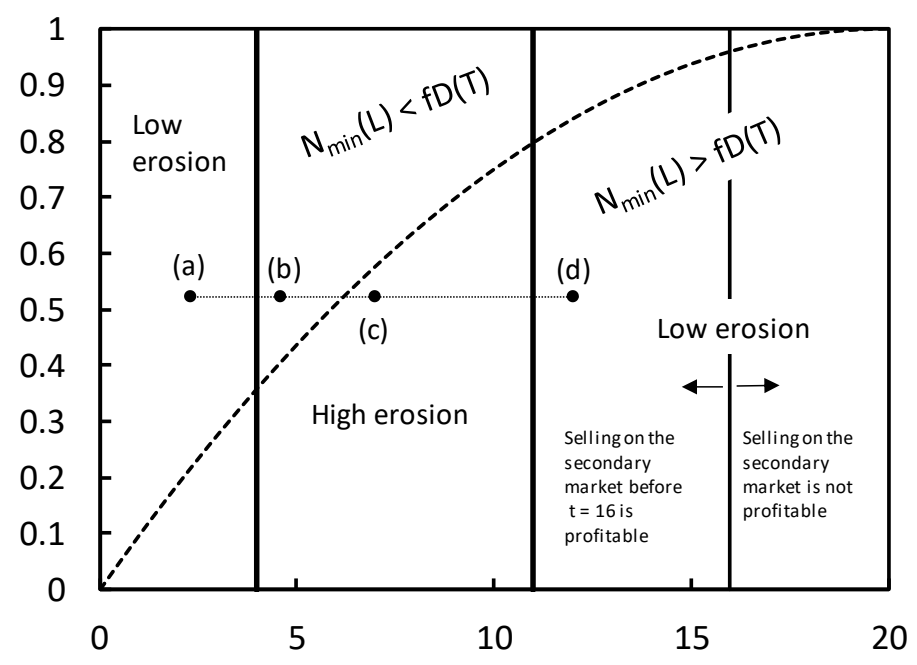

Figure 9: Policy map for the case example

The dotted line in Figure 9 shows the $N_{\min }(L)=f D(T)$ boundary. Points above this line correspond to scenarios where $N_{\min }(L)$ is less than the market capacity $f D(T)$; points below the line correspond to cases where $N_{\min }(L)$ is larger than the market capacity $f D(T)$ and thus, where scrapping applies. By considering $\Delta p_{S}(t)$ vs. the reuse saving $c_{N}+c_{S}-c_{R}$, two limit values for the demo loan period are found. Demo loan period values below $L=4$ months and above $L=11$ months correspond to low value erosion; $L$-values between 4 and 11 months define high erosion cases. We can also consider the relationship $p_{s}(t)$ vs. $c_{N}$. In this example, selling in the secondary market becomes unattractive after $t=16$. Unless necessary (e.g., when $L>16$ ), we will not use new products after $t=16$ to satisfy demo requests. We have indicated these three boundaries in Figure 9, and we also show our four cases: (a) $L=2.5$ months, (b) $L=4.5$ months, (c) $L=6.5$ months and (d) $L=12$ months, which have clearly different characteristics in terms of erosion and $N_{\min }(L)$ vs. $f D(T)$ relation. The $f$-value is $f=180 / 350=0.514$.

Table 3 summarizes key characteristics of the optimal strategies for the 4 different cases. The time $t_{S}$ is the time when the collections exceed the demo requests. For an isosceles triangular profile 
with maximum at $t=10, N_{\min }(L)$ is based on a symmetric $L / 2$ time window around the maximum, $N_{\min }(L)=\int_{10-L / 2}^{10+L / 2} d(t) d t$.

Table 3: Characteristics and evaluation of four cases

\begin{tabular}{|c|c|c|c|c|c|c|c|c|c|c|c|}
\hline Case & $\mathrm{L}$ & $t_{S}$ & $N_{\min }(L)$ & $t_{f}$ & $\Delta t_{f}$ or $\Delta t$ & $N$ & $\begin{array}{c}\text { revenue } \\
(\mathrm{k} \$)\end{array}$ & $\begin{array}{c}\text { cost new } \\
(\mathrm{k} \$)\end{array}$ & $\begin{array}{c}\text { profit } \\
(\mathrm{k} \$)\end{array}$ & $\begin{array}{c}\text { profit }(\mathrm{k} \$) \\
\text { max reuse }\end{array}$ & $\begin{array}{c}\text { profit }(\mathrm{k} \$) \\
\text { no reuse }\end{array}$ \\
\hline$(\mathrm{a})$ & $\mathrm{L}=2.5$ & 11.25 & 82.03 & 9.98 & & 180 & 706.5 & 432 & 274.5 & -4.8 & -33.8 \\
\hline$(\mathrm{b})$ & $\mathrm{L}=4.5$ & 12.25 & 139.78 & 9.29 & 1.71 & 213.72 & 640.1 & 512.9 & 127.2 & -88.5 & -174.8 \\
\hline$(\mathrm{c})$ & $\mathrm{L}=6.5$ & 13.25 & 190.53 & 24.05 & 4.5 & 225.97 & 511.3 & 542.3 & -31 & -77.3 & -287.8 \\
\hline (d) & $\mathrm{L}=12$ & 16 & 294 & 23.93 & & 294 & 343.1 & 705.6 & -362.5 & -362.5 & -457.7 \\
\hline
\end{tabular}

In all cases $p_{S}(t)$ drops below $c_{N}$ after $t^{*}=16$. Low erosion case (a) has $N_{\min }(L)=82.03<f D(T)$ $=180$ and should be controlled according to policy 4.2.(b). The $t_{f}$ value (calculated via Eq.(10)) is well below $t^{*}(9.98<16)$ so that this case reduces to policy 4.1.(b): a total of 180 new products are used to satisfy all demo request. Reuse is postponed until $t_{f}=9.98$; all requests are satisfied by new products and all available collections are sold in the secondary market. Between $t_{f}$ and $t_{s}$, all collections are reused; the demand in excess of the available collections is satisfied by new products, and there are no secondary market sales. Finally, between $t_{S}$ and $T$, all demo requests are satisfied through reuse, and collections in excess of the demo demand are salvaged in the secondary market until $T+L$. There is no scrapping in this case. The flows over time for case (a) are shown in the two diagrams at the top in Figure 10. The left diagram shows reuse $X_{R}(t)$ and demo requests satisfied by new products $X_{N}(t)$; the right diagram shows the collections $(d(t-L))$ and the collections sold in the market $X_{\mathrm{S}}(t)$. The optimal case (a) policy generates a profit of $\$ 274.5 \mathrm{k}$ ( $=$ revenue $\$ 706.5 \mathrm{k}-$ new product $\operatorname{cost}\left(c_{N} * N=\$ 2.4 \mathrm{k} * 180\right.$ $=\$ 432 \mathrm{k})$. The last two columns in Table 3 report the profit of two alternative policies: the max reuse policy uses only $N_{\min }(L)$ new products to satisfy all requests and sells excess collections, up to the market capacity, as soon as possible (i.e., between $t_{S}$ and $T+L$, see the second part of the $X_{M}(\mathrm{t})$ curve in Figure 10 , case (a)). The no reuse policy satisfies each request by a new product (new product cost $\$ 2.4 * 350$ $=\$ 840 \mathrm{k})$, and sells the $f D(T)=180$ earliest collections. Both these policies are quite different from the optimal case (a) policy and generate a loss ( $-\$ 4.8 \mathrm{k}$ and $-\$ 33.8)$. 

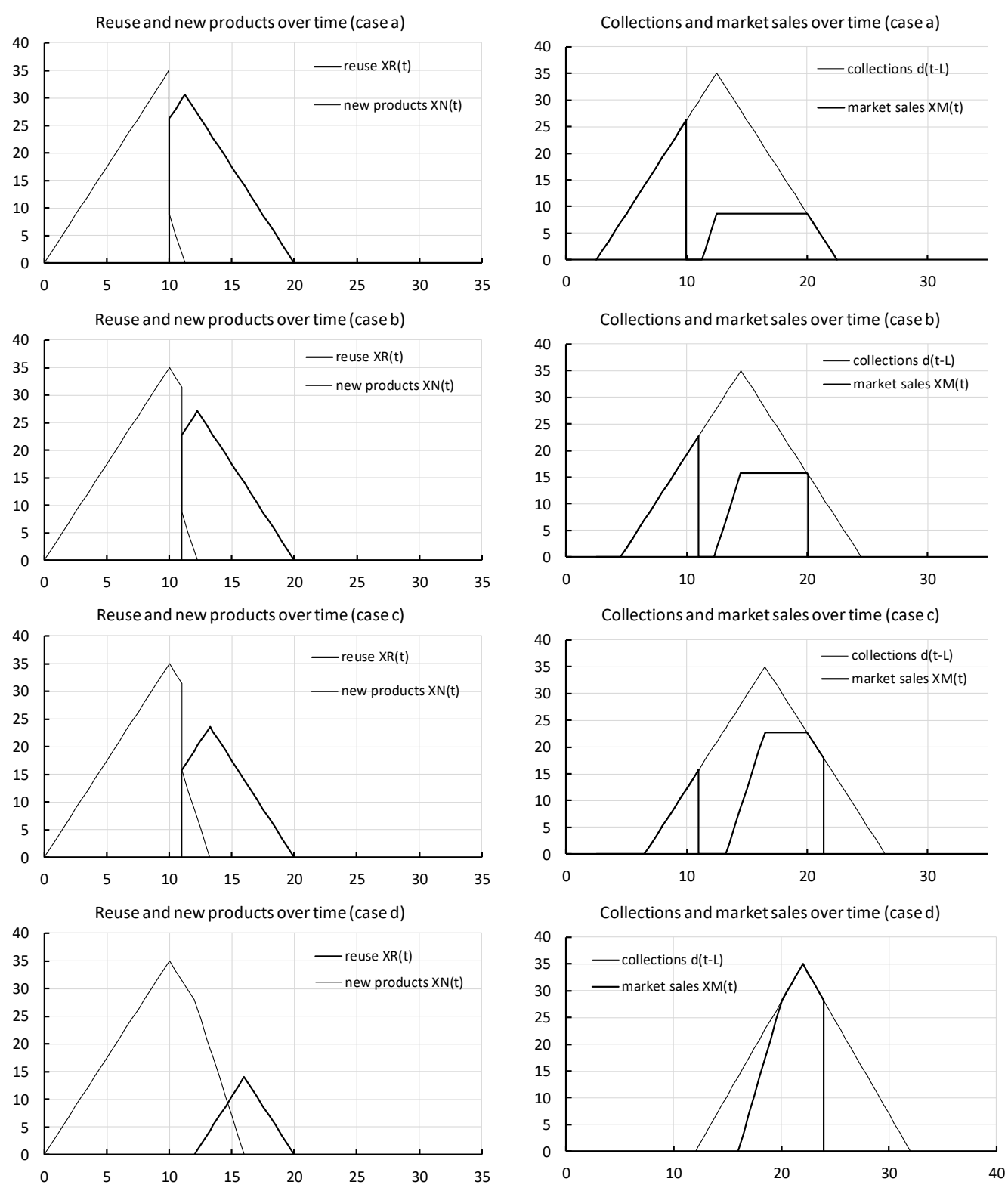

Figure 10: Time flows for the different cases

The cases (b) and (c) are high erosion cases: case (b) with $N_{\min }(L)=139.78<180=f D(T)$ and case (c) with $N_{\min }(L)=190.53>180=f D(T)$. Case (b) is controlled with policy 5.2(b) and case (c) with policy 5.2.(a). For case (b), $t_{f}=9.29$ (calculated via Eq.(10)) and for case (c), $t_{f}=24.05$ (calculated via Eq.(9)). For case (b), the maximum $\Delta t_{f}$ value is found from the $\Delta p_{S}(t)$ function for $L=4.5$, where $t$ $=11$ still shows a value larger than the reuse saving. We set $\Delta t_{f}=11-9.29=1.71$. Also, for case (c), the maximum $\Delta t$ value is determined by $t=11$ in the $\Delta p_{S}(t)$ function for $L=6.5$. In this case, we set $L+\Delta t=11$, so $\Delta t=11-6.5=4.5$. The positive values for $\Delta t$ and $\Delta t_{f}$ clearly show that reuse is further postponed until $t=11$ in the case of high erosion. This means that extra new products are required to satisfy all demo requests: for case (c): $225.97-190.53=35.44$ products (this is in addition to $N_{\min }(L)$ $=190.53$, and the extra amount of new products $35.44<180$ market capacity); for case (b): $213.72-$ 
$180=33.73$ products (this is in addition to $f D(T)=180$, and the extra amount of new products $33.73<$ $139.78=N_{\min }(L)=$ 'reduced' market capacity). The value $t^{*}=16$ is well above $t_{f}+\Delta t_{f}=11$ (case (c)) and $L+\Delta t=11$ (case (b)) and does not impact on the policies.

The flows over time for cases (b) and (c) are shown in the middle section of Figure 10. The curves for case (b) and (c) show a similar but somewhat shifted pattern. We note that for both cases reuse $X_{R}(t)$ is postponed until $t=11$. New products are ordered until $t_{S}=12.25$ (case (b)) and $t_{S}=13.25$ (case (c)); market sales are clearly higher earlier in the lifecycle and also start earlier in case (b); there are no market sales between $t=11$ and $t_{s}$. Both scenarios use more new products than the market capacity and these products are scrapped after the market is saturated. The scrap flows $X_{S}(t)$ are not shown on the left diagrams, but they follow the collection curve near the end of the lifecycle and start when market sales have dropped to zero. From Table 3, we observe that the optimal policy for case (b) yields a profit $(\$ 127.2 \mathrm{k})$, whereas case (c) generates a loss $(-\$ 31 \mathrm{k})$. Both the max reuse and no reuse policies perform much worse and are very costly. By comparing cases (a), (b) and (c), the demo loan period $L$ has a huge impact on the overall profitability and cost effectiveness of the operations. A two month decrease or increase compared with the current $L$-value for processor 1 could either generate a healthy profit or produce a steep loss.

Case (d) is a low erosion example with a long lead time $L=12, N_{\min }(L)=294>180=f D(t)$, so policy 4.2.(a) applies. We use new products for all demo requests until time $t=L=12$. Reuse starts at $t=12$ with all available collections, but also new products are required for some of the requests until $t_{S}$ $=16$. At $t_{S}=16$, we only satisfy requests through reuse and market sales commence and continue until the market is saturated. Remaining collections are scrapped. The flows for case (d) are shown in the bottom part of Figure 10, and are a little different from the other three cases. Table 3 shows that case (d) yields a loss (-\$362.5k) and that the no-reuse policy is worse.
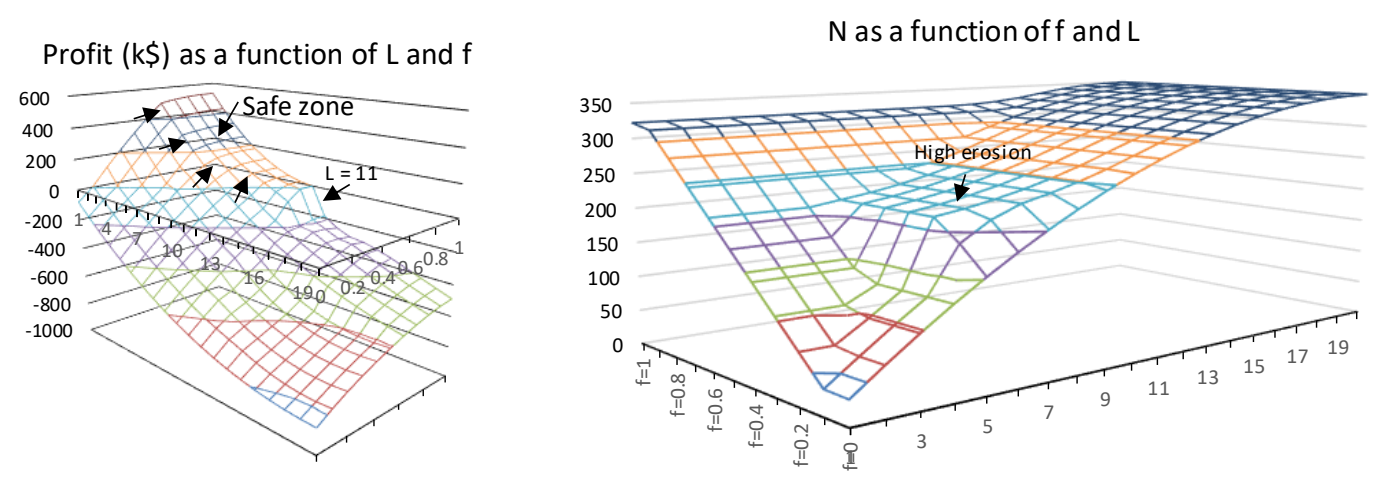

Figure 11: Profit (k\$) and $N$ as a function of $L$ and $f$

Figure 11 shows for processor 1 the total profit $(\mathrm{k} \$$ ) (left) and the number of new products $N$ used to fulfil all demo request (right) for the optimal demo policies as a function of the loan period $L$ (values from 1 to 20 ) and the market capacity ( $f$ values from 0.1 to 1 ). The profit diagram shows that the operations are only profitable when the demo loan period is short, and when the secondary market is 
well developed. Otherwise, profit can erode rapidly and the entire demo process becomes a very costly marketing approach.

The profit diagram reveals key changes in sensitivities, in particular for $L$-values around 11 (when the drop in revenue occurs) and around $f=0.5-0.6$ values for small $L$ and gradually increasing to larger $f$ values when $L$ increases as indicated by the small arrows in Figure 11. The latter is again due to the steep drop in sales revenue at $t=11$ : for low $L, N_{\min }(L)$ is small and in case the market is well developed, the $N=f D(T)>N_{\min }(L)$ policy applies with most sales before $t=11$. A larger $L$, requires a better developed market (higher $f$ ) but there is also less opportunity to sell before $t=11$, so overall profit decreases. The diagram on the right showing $N$ as a function of $f$ and $L$ displays a smoother impact of increasing $L$ on $N$ for constant $f$, especially for larger $L$. This is due to the shape of the demand profile and $N_{\min }(L)$ (see also Figures 6 and 9 showing a smooth concave increasing pattern). For small $L, N$ increases linearly with $f$ (because of the $N=f D(t)$ policy) and reduces a little for high $f$ (because no new products are used after $t^{*}=16$ ). For larger $L, N$ is less sensitive to changes in $f$ (because $N_{\text {min }}(L)>f D(T)$ and some of the collections are scrapped). The high erosion region, where more new products are used, is clearly visible in the diagram.

The optimal flow patterns in Figure 10 show that even for relatively simple demand profiles, product flows over the lifecycle can be quite different and are heavily influenced by lead time. This information should appeal to managers. The summary of policies in Figure 9 provide a clear informative guide to managers on when different policies should be used. Furthermore, constructing flow diagrams like in Figure 10 provide a visual aid, which managers can use to better forecast product returns (collections) and plan and coordinate reuse and resale operations. These diagrams show that we need to evaluate and consider all the relevant characteristics and information to identify an optimal policy. Making a judgement based on partial information (e.g., only considering $p_{S}(t)$ vs. $c_{N}$ and ignoring factors such a demo loan time $L$, market capacity $f$ and the shape of the demo request profile $d(t)$ ), will result in incorrect decisions, which can be very costly for high-end IT product demonstrations. Due to confidentiality, we cannot report exact estimates of savings by the case company in implementing the optimal strategies. However, for products like processor 1, life cycle savings well in excess of $\$ 100 \mathrm{k}$ can be easily achieved with very modest changes (e.g., limiting the demonstration time to 3 months), whereas implementing an optimal strategy could generate savings in the region of $\$ 200 \mathrm{k}-\$ 250 \mathrm{k}$. Processor 1 is only one component from the case companies' product portfolio, and from Table 1, its actual average demo loan time (132 days) compared 'favorably' against other processors with demonstration times between 165 and 220 days. Such excessive demonstration times do not allow much opportunity for the reuse option, and indeed as evidenced from Table 1, 80\% to 90\% of the demo request are satisfied through new products. The no-reuse benchmark policy in Table 3, was however the worst performing policy.

Finally, managers can combine the policy map in Figure 9 with a profit/cost surface plot in Figure 11. This can reveal for different demand and price erosion profiles where the key sensitivities 
in profit are, and what policy changes are required to either drive performance improvement or to identify 'safe zones' (see Figure 11 left). Managers should aim to operate well within a safe zone where small deviations in $L$ and $f$ would still generate a healthy profit.

\section{Conclusions and further research}

In this paper we discussed and investigated a closed-loop supply chain for providing demonstrations of expensive, high-end products, with a short lifecycle. Potential customers request demos, while the manufacturer has the option to satisfy the requests either by new products or by reusing previously collected demos. Ex-demo equipment can be sold in the secondary market, but the product's sales price erodes over time. Eroding sales prices may change the economics of reuse versus no-reuse (resale) during the lifecycle.

We derived two measures, which are necessary to develop an optimal demo strategy in a deterministic setting with concave or unimodal demo demand profile over the product lifecycle. The first indicator is whether or not the sales price $p_{S}(t)$ drops below the acquisition cost $c_{N}$. In general, the time when the sales price drops below $c_{N}$ is a signal to start reuse. The second indicator is whether the drop in sales price over the demo loan time $L$ is larger than the reuse saving $c_{N}+c_{S}-c_{R}$. Large drops in sales prices are characteristics of fast value erosion; small drops in sales price characterize slow value erosion. Inspecting the $\Delta p_{s}(t)$ function provides a convenient way to identify high erosion. We show that the distinction between high and low erosion depends on the stage in the product's lifecycle and the demo loan period $L$. In case of fast erosion, we also show that it may be better to postpone reuse until later in the lifecycle, even if this results in more products being scrapped. The optimal strategy depends on the interactions of several indicators (i.e., if these are present) and on the availability of collected products (residence time $L$ ); the demo demand profile $(d(t))$ and the secondary market potential $(f D(T))$.

The application of the generic analysis presented in this paper led to a number of useful insights for managers at the case company. Managers could recognize the importance of the demo request profile, the value of time, and their effect on value recovery. It became evident also that not all products should be treated in the same way: company $\mathrm{X}$ has products in its portfolio that belong to the four categories considered, and our analysis clearly shows that different optimal policies apply. Furthermore, by applying the generic analysis, managers became aware that more stringent controls and measures were required to manage the demo store, with respect to the demo loan period $L$. Most of the products examined had average loan periods in excess of the recommended 3 months. The longer $L$, the more new products are required to satisfy demo request and the lower the value recovery through reuse and/or resale in the secondary market.

The context of our study focuses on demonstrations of high-end IT products. Demonstrations of medical equipment (e.g., dental chair), optical, audio and measurement equipment and categories of 
industrial equipment and machinery share many of the characteristics above although in some cases the product's lifecycle may be longer. For products with longer lifecycles, it may be recommended to incorporate an additional discount factor to compare cash flows at different times and calculate a net present value. The indicators in the models above can be easily modified for this situation. For example, assuming that $f(t)$ is a decreasing discount function over time and that all cash in/out flows are discounted, it is not too difficult to show that the first signal $\left(p_{s}(t)\right.$ vs. $\left.c_{N}\right)$ is unaffected by the presence of the discount function. Hence, all our results still hold for the low erosion case, but clearly total profit/or cost will be discounted. The second indicator, however, is affected by the discount factor and becomes: $p_{s}(t)-p_{s}(t+L) f(t+L) / f(t)$ vs. $c_{N}+\left(c_{S}-c_{R}\right) f(t+L) / f(t)$. Because $0<f(t+L) / f(t)<1$, the discount factor amplifies the revenue price drop $\Delta p_{s}(t)$. The effect on the reuse saving depends on the relation between $c_{S}$ and $c_{R}$ : if $c_{S}-c_{R}<0$, also the reuse saving is amplified, otherwise it is decreased. The reuse saving part becomes time dependent, rather than static which may change the high/low erosion decision (using $\Delta p_{s}(t)$ function). However, the same generic approach presented in the paper can be used to further explore this situation.

Value erosion is also important other contexts: in the mobile phone sector with new generation handsets introduced every 6 months, and in the fashion/clothing industry where increased online sales go together with increased product returns. Returning, repacking, re-labelling and reselling fashion apparel in a short time window puts tremendous pressure on clothing companies, yet some stores do offer free returns. Elements of the modelling approach we present can be used to capture several key profitability drivers for closed-loop supply chains where the rotation of assets and their subsequent recovery in the secondary market are important. Certain aspects of the model are more generally applicable (e.g., rental or leasing of products), or could be further extended to incorporate revenue from rental or leasing, or other characteristics. More time varying features and/or more constraints can be considered, but the analysis will be more involved. Further extensions could also investigate more complex demand profiles (which may require inventory) and/or stochastic elements (demo loan period, demo demand, secondary market demand).

Our modelling approach as a continuous time rather than a multiple period optimization problem enabled us to focus on the features that define and drive the optimal solution structure. This modelling approach results in stronger and more general conclusions, which is of importance when delays, time value and dynamic features have to be accounted for. Our model can be transformed into a Continuous time Transportation Problem. For the more general Continuous time Minimum Cost Network Flow Problems - although NP-hard to solve (Klinz and Woeginger, 2004) - optimality and strong duality results were recently derived by Koch and Nasrabadi, (2014) under certain conditions. These models can be explored for generalizations of our problem. 


\section{References}

Anderson, E.A. and A.B. Philpott, 1984. Duality and an algorithm for a class of continuous transportation problems. Mathematics of Operations Research 9(2) 222-231.

Atasu, A. and S. Çetinkaya, 2006. Lot sizing for optimal collection and use of remanufacturable returns over a finite life-cycle. Production and Operations Management 15(4) 473-487.

Atasu, A., Guide, Jr., V.D.G, and L.N. Van Wassenhove, 2008. Product reuse economics in closedloop supply chain research. Production and Operations Management 17(5), 483-496

Blackburn, J., 1991. Time-Based Competition. Business One Irwin, Homewood, IL.

Blackburn, J., Guide, Jr., V.D.G, Souza, G. and L.N. Van Wassenhove, 2004. Reverse supply chains for commercial returns. California Management Review 46(2) 6-22.

Dekker, R., Fleischmann, M., Inderfurth, K. and L.N. Van Wassenhove, eds, 2004, Reverse logistics: Quantitative models for closed-loop supply chains. Springer, Heidelberg.

Difrancesco, R.M., Huchzermeier, A. and D. Schröder, 2018. Optimizing the return window for online fashion retailers with closed-loop refurbishment. Omega 78 (C) 205-221.

Fang, C., Liu, X., Pei, J., Fan, W. and P.M. Pardalos, 2016. Optimal production planning in a hybrid manufacturing and recovering system based on the internet of things with closed loop supply chains, Operational Research 16(3) 543-577.

Geyer, R. and T. Jackson, 2004. Supply loops and their constraints: The industrial ecology of recycling and reuse, California Management Review 46 (2) 55-73.

Geyer, R., Van Wassenhove L.N. and A. Atasu, 2007. The economics of remanufacturing under limited component durability and finite product life cycles. Management Science 53(1) 88-100.

Govidan, K., Soleimani, H. and D. Kannan, 2015. Reverse logistics and closed-loop supply chain: A comprehensive review to explore the future. European Journal of Operational Research 240 (3) 603626.

Govidan, K. and H. Soleimani, 2017. A review of reverse logistics and closed-loop supply chains: a Journal of Cleaner Production focus. Journal of Cleaner Production 142, (1) 371-384.

Guide, Jr., V.D.R., Harrison, T. and L.N. Van Wassenhove, 2003. The challenge of closed-loop supply chains. Interfaces 33(6) 3-6.

Guide, Jr., V.D.R. and L.N. Van Wassenhove, eds, 2003. Business Aspects of Closed-Loop Supply Chains. Carnegie Mellon University Press, Pittsburgh, PA.

Guide, Jr., V.D.R., Muyldermans, L. and L.N. Van Wassenhove, 2005. Hewlett-Packard Company unlocks the value potential from time-sensitive returns. Interfaces 35(4) 281-293

Guide, Jr., V.D.G, Souza, G., Van Wassenhove, L.N. and J. Blackburn, 2006. Time value of commercial returns. Management Science 58(2) 1200-1214.

Guide, Jr., V.D.R. and L.N. Van Wassenhove, 2009. OR Forum - The evolution of closed-loop supply chain research. Operations Research 57(1) 10-18. 
Heiman, A. and E. Muller, 1996. Using demonstrations to increase new product acceptance: controlling demonstration time. Journal of Marketing Research 23, 422-430.

Heiman, A., McWilliams, B. and D. Zilberman, 2001. Demonstrations and money-back guarantees: market mechanisms to reduce uncertainty. Journal of Business Research 54, 71-84.

Kadambala, D.K., Subramanian, N., Tiwari, M.K., Abdulrahman, M. and C. Liu, 2017. Closed loop supply chain networks: Design for energy and time value efficiency. International Journal of Production Economics, 183 (Part B) 382-393.

Klinz, B. and G.J. Woeginger, 2004. Minimum cost dynamic flows: The series-parallel case. Networks 43(3) 153-162.

Koch, R. and E. Nasrabadi, 2014. Flows over time in time-varying networks: Optimality conditions and strong duality. European Journal of Operational Research, 237 (2) 580-589.

Krikke, H., van Harten, A. and P.C. Schuur, 1999. Business Case Océ: Reverse logistics network redesign for copiers. OR Spektrum 21(3) 381-409.

Krikke, H., le Blanc, I. and S. van de Velde, 2004. Product modularity and the design of closed-loop supply chains. California Management Review 46 (2) 23-39.

Kroon L. and G. Vrijens, 1995. Returnable Containers: An example of reverse logistics. International Journal of Physical Distribution \& Logistics Management 25(2) 56-68.

Östlin, J., Sundin, E. and M. Björkman, 2008. Importance of closed-loop relationships for product remanufacturing. International Journal of Production Economics 115(2) 336-348.

Pazoki, M. and W. Abdul-Kader, 2016. Optimal disposition decisions for a remanufacturing system considering time value of products. Computers \& Industrial Engineering, 99, 124-136

Pullan, M.C., 1996. A duality theory for separated continuous linear programs. SIAM Journal on Control and Optimization, 34 (3) 931-965

Ruiz-Benítez, R., Ketzenberg, M. and E.A. van der Laan, 2014. Managing consumer returns in high clockspeed industries. Omega, 44 (43) 54-63.

Sasikumar, P. and G. Kannan, 2009. Issues in reverse supply chain, part III: Classification and simple analysis. International Journal of Sustainable Engineering, 2(1) 2-27. 


\section{Appendix}

We show how our problem can be transformed into a Continuous Transportation Problem (CTP). We also demonstrate the optimality of the solution structures derived in Sections 4 and 5, relying on the strong duality results for the CTP (see e.g., Anderson and Philpott, 1984). We present each identified structure as a solution to the CTP in a transportation tableau format. The rows and columns display the supplies (new products or reuse from store) and demands (demo requests, secondary market and the scrapping) that are active in different time epochs. Let $c_{i j}(t)$ denote the cost function to supply demand $j$ from supply $i$. Based on the cells that characterize the solution, we calculate the dual cost functions $\lambda_{i}(t)$ and $\mu_{j}(t)$ for the supply and demand cells via $\lambda_{i}(t)+\mu_{j}(t)=c_{i j}(t)$. This resembles the reduced cost relationships in static Transportation Problems, but in the CTP the $\lambda_{i}(t)$ and $\mu_{j}(t)$ are functions. Next, we calculate the reduced cost functions $c_{i j}(t)-\lambda_{i}(t)-\mu_{j}(t)$ for the cells that are not used in the solution. When all the reduced cost functions are $\leq 0$, we conclude optimality.

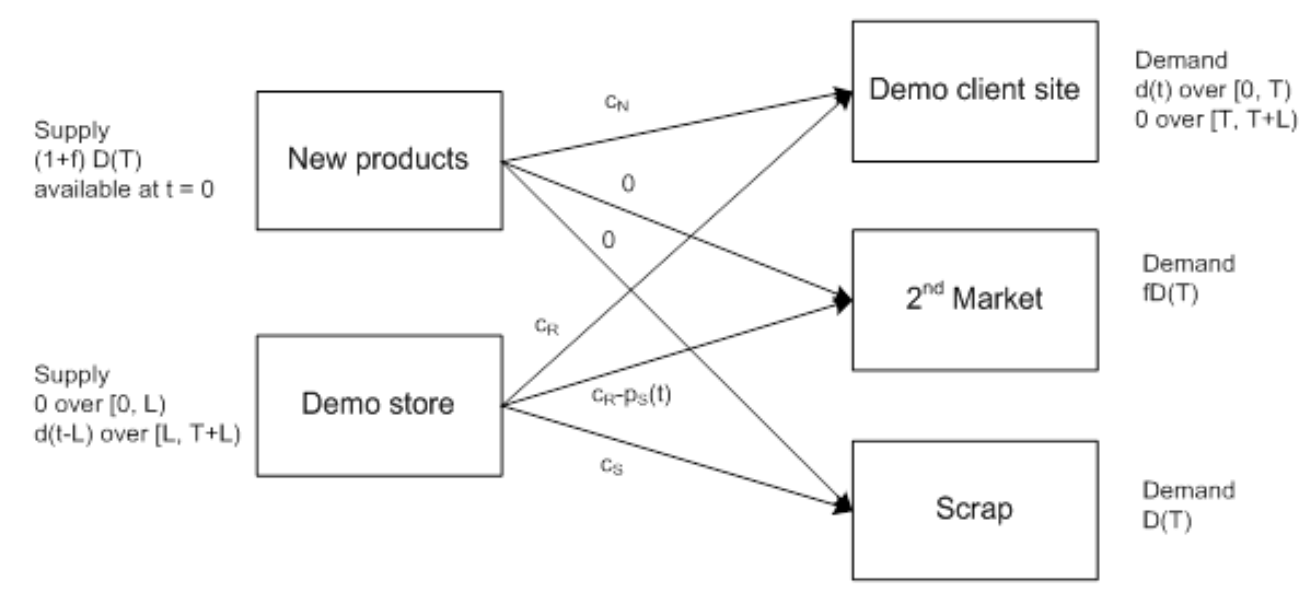

Figure 12: A balanced CTP

Figure 12 shows our problem as a CTP. We model the Demo Store as a supply node (supply of collected ex-demo products) with a time-phased (over loan period $L$ ), time varying supply equal to the time varying demand at the Demo Client Site node. If we specify total demands $f D(T)$ and $D(T)$ for the Secondary Market and Scrap node, then we can set a total supply of $(1+f) D(T)$ at the New Products node to balance the problem. The arcs connecting the New Products node with the Secondary Market and Scrap nodes carry a zero cost. Flows through these two arcs are virtual flows and do not affect the solution. The only arc with a time varying cost is the arc from the Demo Store node to the Secondary Market node, with cost $c_{R}-p_{S}(t)$.

We illustrate the solution structure optimality through two examples. Example 1 (see Section 4.1 and Figure 5(a)) is for the case of low erosion and $p_{S}(t) \geq c_{N}$ over the entire planning horizon $T+L$. We further assume that the minimum number of new products needed to satisfy all demo requests is larger than the secondary market potential $\left(N_{\min }(L)>f D(T)\right.$ ). Example 2 (see Section 5.2 and Figure 7(b)) is for fast erosion $\left(p_{S}(t)\right.$ drops below $c_{N}$ at time $t^{*}$ with $L<t^{*}<T$ and the resale saving $\left(p_{S}(t)+c_{S}\right.$ $\left.-c_{R}\right)$ becomes negative at a time $t_{N}$ with $\left.T<t_{N}<T+L-\Delta t\right)$. In Example 2 we further assume $N_{\min }(L) \leq$ 
$f D(T)$, yet $N=N_{\min }(L)+E\left(t^{*}\right)>f D(t)$. Because the resale saving becomes negative at $t_{N}$, we do not saturate the full market potential but start scrapping at $t_{N}$. We recall that the demo request profile is concave over $[0, T)$ and that the collections follow exactly the same profile, but time shifted over period $L$, i.e., horizon $[L, T+L)$. Further, let $t_{S}$ denote the time when the collections exceed (and stay larger than) the demand. (All input functions are assumed to be piecewise analytic over [0, $T+\mathrm{L}$ ). Piecewise analytic functions are right-continuous but not necessarily left-continuous. They may be discontinuous at a finite number of points.)

Example 1: We examine the case where $t_{S}<t_{f}<T$ (Figure 5(a) shows the example where $t_{S}<T<t_{f}$ ) and identify the epochs where the solution structure changes as follows:

$[0, L)$ : use new products for all requests (no collections available);

$\left[L, t_{S}\right)$ : reuse the collections and use new products to satisfy the remaining demand;

$\left[t_{s}, t_{f}\right)$ : reuse the collections and sell excess collections in the secondary market;

$\left[t_{f}, T\right)$ : reuse the collections and scrap excess collections;

$[T, T+L)$ : scrap the collections.

\begin{tabular}{|c|c|c|c|c|c|c|c|}
\hline & $\begin{array}{c}\text { Demo } \\
\text { requests } \\
{[0, L)}\end{array}$ & $\begin{array}{c}\text { Demo } \\
\text { requests } \\
{\left[L, t_{S}\right)}\end{array}$ & $\begin{array}{c}\text { Demo } \\
\text { requests } \\
{\left[t_{S}, t_{f}\right)} \\
\end{array}$ & $\begin{array}{c}\text { Demo } \\
\text { requests } \\
{\left[t_{f}, T\right)} \\
\end{array}$ & $2^{\text {nd }}$ Market & Scrap & \\
\hline New & $\boldsymbol{d}_{\boldsymbol{l}}{ }^{c_{N}}$ & $\boldsymbol{d}_{2}-\boldsymbol{s}_{\boldsymbol{1}}{ }^{c_{N}}$ & $0_{0} \quad c_{N}$ & ${ }_{0} \quad c_{N}$ & 0 & $\begin{array}{c}c^{0} \\
(\mathbf{1}+f) D(T)- \\
N_{\min }(\boldsymbol{L})\end{array}$ & $\begin{array}{c}(1+f) D(T) \\
\lambda_{1}=0\end{array}$ \\
\hline $\begin{array}{l}\text { Store } \\
{\left[L, t_{S}\right)}\end{array}$ & $\infty$ & $s_{1}$ & $0 \quad c_{R}$ & $0 \quad c_{R}$ & $0^{-p_{1}+c_{R}}$ & $c_{0} \quad c_{S}$ & $\begin{array}{c}s_{I} \\
\lambda_{2}=c_{R^{-}} c_{N}\end{array}$ \\
\hline $\begin{array}{l}\text { Store } \\
{\left[t_{s}, t_{f}\right)}\end{array}$ & $\infty$ & $\infty$ & $c_{R}$ & $c_{R}$ & $\begin{array}{l}-p_{2}+c_{R} \\
\boldsymbol{s}_{2}-\boldsymbol{d}_{3}\end{array}$ & $0 \quad c_{S}$ & $\begin{array}{c}s_{2} \\
\lambda_{3}=p_{3}-p_{2}+c_{S}\end{array}$ \\
\hline $\begin{array}{l}\text { Store } \\
{\left[t_{f}, T\right)}\end{array}$ & $\infty$ & $\infty$ & $\infty$ & $\begin{array}{ll} & c_{R} \\
d_{4} & \end{array}$ & $0^{-p_{3}+c_{R}}$ & $\boldsymbol{s}_{3}-d_{4} \quad c_{S}$ & $\begin{array}{c}s_{3} \\
\lambda_{4}=c_{S}\end{array}$ \\
\hline $\begin{array}{c}\text { Store } \\
{[T, T+L)}\end{array}$ & $\infty$ & $\infty$ & $\infty$ & $\infty$ & $0^{-p_{4}+c_{R}}$ & $s_{4}$ & $\begin{array}{c}s_{4} \\
\lambda_{5}=c_{S}\end{array}$ \\
\hline & $\begin{array}{c}d_{l} \\
\mu_{l}=c_{N}\end{array}$ & $\begin{array}{c}d_{2} \\
\mu_{2}=c_{N}\end{array}$ & $\begin{array}{c}d_{3} \\
\mu_{3}=p_{2^{-}} \\
p_{3}+c_{R^{-}} c_{S}\end{array}$ & $\begin{array}{c}d_{4} \\
\mu_{4}=c_{R^{-}-c_{S}}\end{array}$ & $\begin{array}{c}f D(T) \\
\mu_{5}=-p_{3}+c_{R^{-}} \\
c_{S}\end{array}$ & $\begin{array}{l}D(T) \\
\mu_{6}=0\end{array}$ & \\
\hline
\end{tabular}

Figure 13: Optimal CTP solution structure for Example 1.

Figure 13 shows the CTP for Example 1 in a transportation tableau format. We have suppressed the time dependency in the notation: the demo store supply functions are $s_{1}, s_{2}, s_{3}$ and $\mathrm{s}_{4}$, the demo request functions $d_{1}, d_{2}, d_{3}$ and $d_{4}$ and the secondary market price functions $p_{1}, p_{2}, p_{3}$ and $p_{4}$. In the top right corner in each cell we show the relevant cost function. The cells with shaded borders fully characterize a feasible solution to the CTP. With $m$ supply cells and $n$ demand cells we need $m+n-1$ cells that do not contain a loop. In this example the CTP solution is degenerate: we need 5+6-1 = 10 
cells and these are all the cells with positive flow and cell $(4,5)$ with zero flow. Note that $d_{1}+d_{2}+d_{3}+d_{4}=s_{1}+s_{2}+s_{3}+s_{4}$ and $d_{1}+d_{2}-s_{1}=s_{3}+s_{4}-d_{4}=N_{\min }(L)$ so that it is easily verified that the proposed solution structure is feasible. The dual cost functions $\lambda_{i}(t)$ and $\mu_{j}(t)$ are shown in the last column and bottom row; we arbitrarily set $\lambda_{I}(t)=0$.

We calculate the reduced cost functions $c_{i j}(t)-\lambda_{i}(t)-\mu_{j}(t)$ for the cells $(1,3),(1,4),(1,5),(2$, $3),(2,4),(2,5),(2,6),(3,4),(3,6)$ and $(5,5)$. If any of these would price out negatively, we conclude that the solution structure is not optimal; otherwise it is optimal.

\section{Cell $(1,3)$ :}

$c_{13}-\lambda_{1}-\mu_{3}<0$

$c_{N}-0-\left(p_{2}-p_{3}+c_{R}-c_{S}\right)<0$

$c_{N}+c_{S}-c_{R}<p_{2}-p_{3}$

No, because there are no large price drops.

Cell $(1,4)$ :

$c_{14}-\lambda_{1}-\mu_{4}<0$

$c_{N}-0-\left(c_{R}-c_{S}\right)<0$

$c_{N}+c_{S}-c_{R}<0$

No, because the reuse saving is positive.

\section{Cell $(1,5):$}

$c_{15}-\lambda_{1}-\mu_{5}<0$

$0-0-\left(-p_{3}+c_{R}-c_{S}\right)<0$

$p_{3}<c_{S}-c_{R}$

No, because $p_{3}>c_{N}$ (selling is profitable) and $c_{N}+c_{S}-c_{R}>0$

Cell $(2,3):$ (reuse saving), so $p_{3}>c_{N}>c_{S}-c_{R}$.

$c_{23}-\lambda_{2}-\mu_{3}<0$

$c_{R}-\left(c_{R}-c_{N}\right)-\left(p_{2}-p_{3}+c_{R}-c_{S}\right)<0$

$c_{N}+c_{S}-c_{R}<p_{2}-p_{3}$

No, because there are no large price drops.

Cell $(2,4)$ :

$c_{24}-\lambda_{2}-\mu_{4}<0$

$c_{R}-\left(c_{R}-c_{N}\right)-\left(c_{R}-c_{S}\right)<0$

$c_{N}+c_{S}-c_{R}<0$

No, because the reuse saving is positive.

Cell $(2,5)$ :

$c_{25}-\lambda_{2}-\mu_{5}<0$

$-p_{1}+c_{R}-\left(c_{R}-c_{N}\right)-\left(-p_{3}+c_{R}-c_{S}\right)<0$

$c_{N}+c_{S}-c_{R}<p_{1}-p_{3}$

No, because there are no large price drops.

\section{Cell $(2,6):$}

$c_{26}-\lambda_{2}-\mu_{6}<0$

$c_{S}-\left(c_{R}-c_{N}\right)-0<0$

$c_{N}+c_{S}-c_{R}<0$

No, because the reuse saving is positive.

Cell $(3,4)$ :

$c_{34}-\lambda_{3}-\mu_{4}<0$

$c_{R}-\left(p_{3}-p_{2}+c_{S}\right)-\left(c_{R}-c_{S}\right)<0$

$p_{2}-p_{3}<0$

No, because $p$ is non-increasing. 
Cell $(3,6)$ :

$c_{36}-\lambda_{3}-\mu_{6}<0$

$c_{S}-\left(p_{3}-p_{2}+c_{S}\right)-0<0$

$p_{2}-p_{3}<0$

No, because $p$ is non-increasing.

Cell $(5,5):$

$C_{55}-\lambda_{5}-\mu_{5}<0$

$-p_{4}+c_{R}-c_{S}-\left(-p_{3}+c_{R}-c_{S}\right)<0$

$p_{3}-p_{4}<0$

No, because $p$ is non-increasing.

We conclude that the solution structure is optimal.

Example 2: The epochs where the solution structure changes are as follows (Section 5.2):

$[0, L)$ : use new products for all requests (no collections available);

$\left[L, t^{*}\right)$ : use new products for all requests, sell all collections in the secondary market;

$\left[t^{*}, T\right)$ : reuse the collections for all requests and sell excess collections in the secondary market;

$\left[T, t_{N}\right)$ : sell all collections in the secondary market;

$\left[t_{N}, T+L\right)$ : scrap all collections.

Figure 14 shows the CTP for Example 2 in a transportation tableau format. It is readily verified that the cells with positive flow $(5+5-1=9$ in total $)$ are a feasible CTP solution.

\begin{tabular}{|c|c|c|c|c|c|c|}
\hline & $\begin{array}{c}\text { Demo } \\
\text { requests } \\
{[0, L)}\end{array}$ & $\begin{array}{l}\text { Demo } \\
\text { requests } \\
{\left[L, t^{*}\right)}\end{array}$ & $\begin{array}{c}\text { Demo } \\
\text { requests } \\
{\left[t^{*}, T\right)} \\
\end{array}$ & $2^{\text {nd }}$ Market & Scrap & \\
\hline New & $\begin{array}{ll}\boldsymbol{d}_{\boldsymbol{l}} & c_{N}\end{array}$ & $\begin{array}{ll}\boldsymbol{d}_{2} & c_{N}\end{array}$ & $0 c_{N}$ & $\begin{array}{c}0 \\
f D(T)-s_{1}-s_{2-} \\
s_{3}+d_{3}\end{array}$ & $\boldsymbol{D}(\boldsymbol{T})-\mathbf{s} 4$ & $\begin{array}{c}(1+f) D(T) \\
\lambda_{I}=0\end{array}$ \\
\hline $\begin{array}{l}\text { Store } \\
{\left[L, t^{*}\right)}\end{array}$ & $\infty$ & $\overline{c_{R}}$ & $c_{R}$ & $s^{-p_{l}+c_{R}}$ & $c_{S}$ & $\begin{array}{c}s_{1} \\
\lambda_{2}=c_{R}-p_{1}\end{array}$ \\
\hline $\begin{array}{l}\text { Store } \\
{\left[t^{*}, T\right)}\end{array}$ & $\infty$ & $\infty$ & $\begin{array}{ll} & c_{R} \\
d_{3} & \end{array}$ & $\begin{array}{l}-p_{2}+c_{R} \\
\boldsymbol{s}_{2}-\boldsymbol{d}_{3}\end{array}$ & $c_{S}$ & $\begin{array}{c}s_{2} \\
\lambda_{3}=c_{R}-p_{2}\end{array}$ \\
\hline $\begin{array}{l}\text { Store } \\
{\left[T, t_{N}\right)}\end{array}$ & $\infty$ & $\infty$ & $\infty$ & $s_{3}^{-p_{3}+c_{R}}$ & $c_{S}$ & $\begin{array}{c}s_{3} \\
\lambda_{4}=c_{R}-p_{3}\end{array}$ \\
\hline $\begin{array}{c}\text { Store } \\
{\left[t_{N}, T+L\right)}\end{array}$ & $\infty$ & $\infty$ & $\infty$ & $0^{-p_{4}+c_{R}}$ & $S_{4}$ & $\begin{array}{c}s_{4} \\
\lambda_{5}=c_{S}\end{array}$ \\
\hline & $\begin{array}{c}d_{1} \\
\mu_{1}=c_{N}\end{array}$ & $\begin{array}{c}d_{2} \\
\mu_{2}=c_{N}\end{array}$ & $\begin{array}{c}d_{3} \\
\mu_{3}=p_{2}\end{array}$ & $\begin{array}{l}f D(T) \\
\mu_{4}=0\end{array}$ & $\begin{array}{c}D(T) \\
\mu_{5}=0\end{array}$ & \\
\hline
\end{tabular}

Figure 14: Optimal CTP solution structure for Example 2.

We calculate the reduced cost functions $c_{i j}(t)-\lambda_{i}(t)-\mu_{j}(t)$ for the cells $(1,3),(2,2),(2,3),(2,5),(3$, $5),(4,5)$ and $(5,4)$ : 
Cell $(1,3)$ :

$c_{13}-\lambda_{1}-\mu_{3}<0$

$c_{N}-0-p_{2}<0$

$c_{N}<p_{2}$

No, $p$ drops below $c_{N}$ at time $t^{*}$.

Cell $(2,2)$ :

$c_{22}-\lambda_{2}-\mu_{3}<0$

$\left.c_{R}-\left(-p_{1}+c_{R}\right)-c_{N}\right)<0$

$p_{1}<c_{N}$

No, $p$ doesn't drops below $c_{N}$ until time $t^{*}$.

Cell $(2,3)$ :

$c_{23}-\lambda_{2}-\mu_{3}<0$

$c_{R}-\left(-p_{1}+c_{R}\right)-p_{2}<0$

$p_{1}-p_{2}<0$

No, because $p$ is non-increasing.

Cell $(2,5)$ :

$c_{25}-\lambda_{2}-\mu_{5}<0$

$c_{S}-\left(-p_{1}-c_{R}\right)-0<0$

$p_{1}+c_{S}-c_{R}<0$

No, because the resale saving is positive until $t_{N}$.

Cell $(3,5)$ :

$c_{35}-\lambda_{3}-\mu_{5}<0$

$c_{S}-\left(-p_{2}-c_{R}\right)-0<0$

$p_{2}+c_{S}-c_{R}<0$

No, because the resale saving is positive until $t_{N}$.

Cell $(4,5)$ :

$c_{45}-\lambda_{4}-\mu_{5}<0$

$c_{S}-\left(-p_{3}-c_{R}\right)-0<0$

$p_{3}+c_{S}-c_{R}<0$

No, because the resale saving is positive until $t_{N}$.

Cell $(5,4):$

$c_{54}-\lambda_{5}-\mu_{4}<0$

$\left(-p_{4}-c_{R}\right)-c_{S}-0<0$

$p_{4}+c_{S}-c_{R}>0$

No, because the resale saving becomes negative at $t_{N}$.

Because none of the cells price out favorably, we conclude optimality. The same procedure can be applied to prove optimality of the different solution structures in Sections 4 and 5. 